\title{
Circadian Rhythms of Perineuronal Net Composition
}

\author{
DHarry Pantazopoulos, ${ }^{1}$ Barbara Gisabella, ${ }^{1}$ Lindsay Rexrode, ${ }^{1}$ David Benefield, ${ }^{1}$ Emrah Yildiz, ${ }^{2}$ \\ Phoebe Seltzer, ${ }^{2}$ Jake Valeri, ${ }^{1}$ Gabriele Chelini, ${ }^{2,3}$ Anna Reich, ${ }^{2}$ Magdalena Ardelt, ${ }^{5}$ and \\ (1) Sabina Berretta $2,3,4$
}

\section{https://doi.org/10.1523/ENEURO.0034-19.2020}

${ }^{1}$ University of Mississippi Medical Center, Jackson, MS 39216, ${ }^{2}$ Translational Neuroscience Laboratory, Mclean Hospital, Belmont, MA 02478, ${ }^{3}$ Department of Psychiatry, Harvard Medical School, Boston, MA 02215, ${ }^{4}$ Program in Neuroscience, Harvard Medical School, Boston, MA 02115, and ${ }^{5}$ Ralph H. Johnson VA Medical Center, Charlestown, SC 29406

\begin{abstract}
Perineuronal nets (PNNs) are extracellular matrix (ECM) structures that envelop neurons and regulate synaptic functions. Long thought to be stable structures, PNNs have been recently shown to respond dynamically during learning, potentially regulating the formation of new synapses. We postulated that PNNs vary during sleep, a period of active synaptic modification. Notably, PNN components are cleaved by matrix proteases such as the protease cathepsin-S. This protease is diurnally expressed in the mouse cortex, coinciding with dendritic spine density rhythms. Thus, cathepsin-S may contribute to PNN remodeling during sleep, mediating synaptic reorganization. These studies were designed to test the hypothesis that PNN numbers vary in a diurnal manner in the rodent and human brain, as well as in a circadian manner in the rodent brain, and that these rhythms are disrupted by sleep deprivation. In mice, we observed diurnal and circadian rhythms of PNNs labeled with the lectin Wisteria floribunda agglutinin (WFA+ PNNs) in several brain regions involved in emotional memory processing. Sleep deprivation prevented the daytime decrease of WFA+ PNNs and enhances fear memory extinction. Diurnal rhythms of cathepsin-S expression in microglia were observed in the same brain regions, opposite to PNN rhythms. Finally, incubation of mouse sections with cathepsin$S$ eliminated PNN labeling. In humans, WFA+ PNNs showed a diurnal rhythm in the amygdala and thalamic reticular nucleus (TRN). Our results demonstrate that PNNs vary in a circadian manner and this is disrupted by sleep deprivation. We suggest that rhythmic modification of PNNs may contribute to memory consolidation during sleep.
\end{abstract}

Key words: circadian rhythms; extracellular matrix; memory consolidation; perineuronal nets; psychiatric disorders; sleep

\section{Significance Statement}

The mechanisms underlying memory consolidation are not completely understood. Perineuronal nets (PNNs) are extracellular matrix (ECM) structures enveloping subsets of neurons and are involved in regulating synaptic plasticity. Recent studies indicate that PNNs are modified during learning to allow for formation of new synapses. During sleep, synapses are proposed to undergo modification as memory consolidation processes occur. Furthermore, microglia are involved in synaptic regulation and produce several proteases that cleave PNN components. We demonstrate that PNNs are modified in a circadian manner and coincide with expression rhythms of the protease cathepsin-S. These rhythms may contribute to altered synaptic plasticity reported during sleep, suggesting a key process through which proteases modify PNNs to allow for to memory consolidation.

Received January 25, 2019; accepted May 22, 2020; First published July 27, 2020.

The authors declare no competing financial interests.
Author contributions: H.P. and B.G. designed research; H.P., B.G., L.R., D.B., E.Y., P.S., J.V., G.C., A.R., and M.A. performed research; H.P. and B.G. analyzed data; H.P., B.G., and S.B. wrote the paper. 


\section{Introduction}

Perineuronal nets (PNNs) are extracellular matrix (ECM) structures surrounding subpopulations of neurons. PNNs form during the end of critical periods of plasticity, marking their closure by conferring an adult form of restricted plasticity (Pizzorusso et al., 2002; Gogolla et al., 2009; Mauney et al., 2013). Although PNNs have been historically considered stable structures, recent studies suggest they are modified during learning to allow for formation of synapses (Nagy et al., 2007; Brown et al., 2009; Ganguly et al., 2013; Banerjee et al., 2017; Slaker et al., 2018). An important line of evidence comes from studies on matrix metalloproteases, which cleave ECM components including chondroitin sulfate proteoglycans (CSPGs), key components of PNNs, and contribute to regulation of synaptic plasticity (Muir et al., 2002; Porter et al., 2005; Bajor and Kaczmarek, 2013). Expression of these proteases contributes to fear learning and memory consolidation (Brown et al., 2009; Ganguly et al., 2013). These effects are mediated through dendritic spine remodeling (Szklarczyk et al., 2002) and regulation of long-term plasticity (LTP; Nagy et al., 2006). In addition, increasing number of studies directly show that CSPGs, and in turn PNNs, are critically involved in the regulation of synaptic plasticity (Bukalo et al., 2001). Notably, the strength of LTP has been shown to vary in a circadian manner in the hippocampus (Chaudhury et al., 2005), a region where PNN functions in regulating synaptic strength and stability have been particularly well characterized (Bukalo et al., 2001; Brakebusch et al., 2002; Geissler et al., 2013). Together, these observations support the hypothesis that PNN composition may be regulated in a circadian manner to allow for circadian rhythms in synaptic plasticity.

PNN composition is regulated by several cell types including astrocytes and neurons, which produce several of the core PNN components, along with a broad range of endogenous proteases that cleave PNN components produced primarily by astrocytes and microglia (Pantazopoulos and Berretta, 2016; Miyata and Kitagawa, 2017; Bozzelli et al., 2018). We focus on the microglial protease cathepsin-S as a first step toward identifying molecules that may contribute to circadian rhythms in PNN composition. Cathepsin-S has been shown to regulate synaptic plasticity, cleave several ECM components, and is expressed diurnally in the rodent cortex (Petanceska et al., 1996; Hayashi et al., 2013b). Furthermore, several lines of evidence suggest that microglia contribute to diurnal regulation of PNNs. Compelling data point to the role of microglia in the regulation of

This work was supported by the University of Mississippi Medical Center Intramural Research Support Program Research Fund, the National Institutes of Health Grant MH117460, National Institute of Mental Health Grants R01MH091348 and R01 MH105608, and the Phyllis and Jerome Lyle Rappaport Foundation.

Acknowledgements: We thank brain donors and their families for the tissue samples used in these studies.

Correspondence should be addressed to Harry Pantazopoulos at cpantazopoulos@umc.edu.

https://doi.org/10.1523/ENEURO.0034-19.2020

Copyright (C) 2020 Pantazopoulos et al.

This is an open-access article distributed under the terms of the Creative Commons Attribution 4.0 International license, which permits unrestricted use, distribution and reproduction in any medium provided that the original work is properly attributed. synaptic plasticity (Wake et al., 2013; Stevens and Schafer, 2018). Furthermore, microglial dysfunction in the hippocampus results in reduction of dendritic spines along with increased ECM expression (Bolós et al., 2018), suggesting microglia participate in degrading the ECM to allow for increased synaptic plasticity. A recent study demonstrated that pharmacological depletion of microglia prevented PNN decreases that normally occur in a mouse model of Huntington's disease and improved memory function (Crapser et al., 2020), suggesting that microglia play a critical role in regulating $\mathrm{PNN}$ composition. Taken together, the current evidence suggests that cathepsin-S from microglia is an optimal candidate for contributing to modification of PNN composition to allow dynamic regulation of synaptic plasticity during sleep.

PNNs are well represented in neural circuits involved in emotion processing and critically involved in the regulation of fear and reward memories (Gogolla et al., 2009; Slaker et al., 2015; Banerjee et al., 2017; Lasek et al., 2018). Consistent with these observations, PNNs have been implicated in several brain disorders involving these regions, including schizophrenia, bipolar disorder, Alzheimer's disease and addiction (Baig et al., 2005; Morawski et al., 2010; Pantazopoulos et al., 2010, 2015; Mauney et al., 2013; Xue et al., 2014; Slaker et al., 2015; Steullet et al., 2018; Blacktop and Sorg, 2019). Several of these disorders also show altered sleep and circadian rhythms (Lim et al., 2013; McClung, 2013; Wang et al., 2015; Manoach et al., 2016; Pantazopoulos et al., 2017). Thus, diurnal modulation of PNNs has a broad range of implications for psychiatric disorders and memory processing.

We tested the hypothesis that PNNs vary in a circadian manner and that these rhythms are disrupted by sleep deprivation. In mice, we first assessed densities of PNNs across the 24-h cycle in brain regions involved in emotional memory processing and implicated in psychiatric disorders (Vyas et al., 2002; Sartorius et al., 2010; Li et al., 2011; Mahan and Ressler, 2012; Mauney et al., 2013; Meyer et al., 2014; Pantazopoulos et al., 2017; Wells et al., 2017). We then assessed the relationship between PNNs and sleep by testing the effect of sleep deprivation on PNN densities in several regions including the hippocampus, a brain region in which diurnal differences in LTP were reported (Chaudhury et al., 2005).

As a first step in testing the hypothesis that matrix proteases are involved in regulating PNN rhythmicity, we characterized rhythms of cathepsin-S expression. We then demonstrated that cathepsin-S impacts PNN integrity by incubating mouse sections in active cathepsin-S enzyme. Finally, we tested the hypothesis that PNN rhythmicity is conserved in humans, focusing on the amygdala and thalamic reticular nucleus (TRN), two regions in which PNN deficits in schizophrenia and bipolar disorder were reported (Pantazopoulos et al., 2010, 2015; Steullet et al., 2018).

\section{Materials and Methods}

\section{Antibodies and lectin labeling}

Wisteria floribunda agglutinin (WFA)

WFA (catalog \#B-1355, Vector Labs), a lectin isolated from seeds of Wisteria floribunda, binds specifically to $N$ - 
acetyl-D-galactosamine on the terminal end of chondroitin sulfate (CS) chains, with a preference for $\beta$ glycosidic linkage (Kurokawa et al., 1976). The specificity of WFA as a marker for these macromolecules is supported by extensive literature, including ablation of labeling following CS enzymatic digestion (Galtrey and Fawcett, 2007; Pantazopoulos et al., 2010).

\section{Cathepsin-S (E-3)}

Cathepsin-S E-3 (sc-271619, Santa Cruz Biotechnology Inc.) is a mouse monoclonal antibody raised against a peptide matching amino acids $302-331$ at the $\mathrm{C}$ terminus of human cathepsin-S, shown to detect the 24-kDa form of cathepsin-S (sc-271619 data sheet, Santa Cruz Biotechnology Inc.).

\section{IBA1}

IBA1 (019-19 741, FUJIFILM Wako Chemicals USA) is a rabbit polyclonal antibody raised against a synthetic peptide to the $\mathrm{C}$ terminus of IBA1, shown to detect the 17$\mathrm{kDa}$ form of IBA1 in rat and mouse brain samples (01919741 data sheet, FUJIFILM Wako Chemicals USA).

\section{Immunocytochemistry (mouse samples)}

Free-floating tissue sections were carried through antigen retrieval in citric acid buffer $(0.1 \mathrm{~m}$ citric acid and $0.2 \mathrm{M}$ $\mathrm{Na}_{2} \mathrm{HPO}_{4}$ ), heated to $80^{\circ} \mathrm{C}$ for $30 \mathrm{~min}$, and incubated in biotinylated WFA lectin (catalog \#B-1355, Vector Labs) or the mouse monoclonal primary antibody anti-cathepsin-S (1:500, sc-271619, Santa Cruz Biotechnology Inc.) for $48 \mathrm{~h}$, and subsequently in biotinylated secondary antibody (horse anti-goat IgG; 1:500; Vector Labs), followed by streptavidin conjugated with horse-radish peroxidase for $2 \mathrm{~h}(1: 5000 \mu \mathrm{l}$, Zymed), and, finally, in nickel-enhanced diaminobenzidine/ peroxidase reaction $(0.02 \%$ diaminobenzidine, SigmaAldrich, $0.08 \%$ nickel-sulfate, $0.006 \%$ hydrogen peroxide in PB). All solutions were made in PBS with $0.2 \%$ Triton X-100 (PBS-Tx) unless otherwise specified. Immunostained sections were mounted on gelatin-coated glass slides, coverslipped, and coded for blinded quantitative analysis. All sections included in the study were processed simultaneously within the same session to avoid procedural differences. Omission of the primary or secondary antibodies did not result in detectable signal, and preabsorption of mouse anti cathepsin-S with 300 nanograms of active human cathepsin-S (SRP0292, Sigma-Aldrich) did not result in detectable immunolabeling signal.

\section{Dual antigen immunofluorescence}

Sections were co-incubated in primary antibodies (catS, I:500 $\mu$, rabbit anti-IBA1, I:1000 $\mu$; FUJIFILM Wako, catalog \#019-19741); in 2\% bovine serum albumin (BSA) for $72 \mathrm{~h}$ at $4^{\circ} \mathrm{C}$. This step was followed by $4-\mathrm{h}$ incubation at room temperature in Alexa Fluor goat anti-mouse 647 (1:300 $\mu \mathrm{l} ; \mathrm{A}-21235$, Invitrogen) and donkey anti-rabbit 555 (1:300 $\mu \mathrm{l} ; \mathrm{A}-32$ 794, Invitrogen), 10-min incubation in DAPI $1: 16000$ in $0.1 \mathrm{M} \mathrm{PB}$, followed by $10 \mathrm{~min}$ in $1 \mathrm{~mm} \mathrm{CuSO}_{4}$ solution ( $\mathrm{pH} 5.0$ ) to block endogenous lipofuscin autofluorescence (Schnell et al., 1999). Sections were mounted and coverslipped using Dako mounting media (S3023, Dako).

\section{Immunocytochemistry (human samples)}

Free-floating tissue sections were carried through antigen retrieval in citric acid buffer $(0.1 \mathrm{~m}$ citric acid and $0.2 \mathrm{M}$ $\mathrm{Na}_{2} \mathrm{HPO}_{4}$ ), heated to $80^{\circ} \mathrm{C}$ for $30 \mathrm{~min}$, and incubated in biotinylated WFA lectin (catalog \#B-1355, Vector Labs) for $48 \mathrm{~h}$, followed by streptavidin conjugated with horseradish peroxidase for $2 \mathrm{~h}(1: 5000 \mu \mathrm{l}$, Zymed), and, finally, in nickel-enhanced diaminobenzidine/peroxidase reaction $(0.02 \%$ diaminobenzidine, Sigma-Aldrich, $0.08 \%$ nickel-sulfate, $0.006 \%$ hydrogen peroxide in PB). All solutions were made in PBS-Tx unless otherwise specified. Immunostained sections were mounted on gelatincoated glass slides, coverslipped, and coded for blinded quantitative analysis. All sections included in the study were processed simultaneously within the same session to avoid procedural differences. Omission of the WFA lectin or HRP-conjugated streptavidin did not result in detectable signal.

\section{Data collection (mouse)}

In mouse brain samples, serial sections containing the hippocampus, infralimbic cortex, prelimbic cortex, TRN, and habenula were quantified using a Leica microscope interfaced with Bioquant Nova Prime v6.0 (R\&M Biometrics). Borders of each region were defined according to the Allen Brain Atlas and traced under $4 \times$ magnification. Each traced region was systematically scanned through the full $x-, y-$, and $z$-axes under $40 x$ magnification to count each WFA+ PNN or cathepsinS-immunoreactive (IR) microglial cell.

Dual immunofluorescence sections labeled for cathepsin-S and IBA1 from three adult male mice housed in a standard light-dark cycle (four sections per mouse) and killed at zeitgeber time (ZT)6 were quantified using Stereo-Investigator Image Analysis System (v.10.0; MBF Biosciences), interfaced with an Olympus BX-61 microscope. Cathepsin-S-IR cells were distinguished from cathepsin-S-IR blood vessels by the presence or absence of DAPI-stained nuclei.

\section{Data collection (human)}

In human postmortem samples, total numbers and numerical densities of PNNs labeled with WFA were quantified using stereology based sampling (Pantazopoulos et al., 2007; Dorph-Petersen and Lewis, 2011) in the amygdala and TRN in a cohort of postmortem brain samples from human subjects (14 amygdala, 15 TRN subjects). WFA-labeled (WFA+) PNNs were counted in the lateral $(\mathrm{LN})$, basal $(\mathrm{BN})$, accessory basal (AB), and cortical (CO) nuclei of the amygdala, and in TRN using a Zeiss Axioskop-2 Plus interfaced with Stereo-Investigator 6.0 (Microbrightfield Inc.). Intrarater (H.P. and M.A.) reliability of at least $95 \%$ was established before formal data collection and reassessed regularly. The borders of amygdala nuclei were traced and confirmed in adjacent Nisslstained sections according to cytoarchitectonic criteria described previously (Sims and Williams, 1990; Amaral et al., 1992). The nomenclature adopted was used by Sorvari et al. (1995). The central, medial and anterior 
nuclei could not be quantified because their dorso-medial portion was damaged in several samples. The borders of the TRN were identified according to specific landmarks, such as the internal capsule laterally and the subthalamic nucleus ventromedially. Each traced region was systematically scanned through the full $x-, y$-, and $z$-axes to count each WFA-labeled PNN over complete sets of serial sections (6-10 sections) representing the whole extent of the amygdala from each subject (section interval $1040 \mu \mathrm{m}$ ). Outcome measures were plotted by time of death (TOD) for each subject to analyze potential diurnal fluctuations using approaches reported previously in postmortem studies (Monk et al., 1997; Dumont et al., 1999; Zhou et al., 2001; Hofman, 2003; Iwata et al., 2013; Li et al., 2013; Schmal et al., 2013; Pantazopoulos et al., 2017).

\section{Statistical analysis}

Differences between groups relative to the main outcome measures were assessed for statistical significance using stepwise linear regression (ANCOVA). Logarithmic transformation was uniformly applied to all human data values because data were not normally distributed. Statistical analyses were performed using JMP PRO v14 (SAS Institute Inc.). Average daily wheel-running activity was included as a covariate for all mouse studies. TOD was obtained from the death certificate for each subject and tested for potential effects on outcome measures. TOD was also used to divide subjects into subjective day (s-Day TOD, 6 A.M. to 5:59 P.M.) and subjective night (s-Night, 6 P.M. to 5:59 A.M.) groups on the basis of previous literature indicating diurnal fluctuations in the amygdala of humans and mice (Berelowitz et al., 1981; Arnold et al., 1982; Rubinow, 1986). Effects of TOD on outcome measures were analyzed using two steps: (1) subjects were divided into s-Day versus sNight groups for comparisons using stepwise linear regression analysis; and (2) we used quartic regression analysis on plots of $\mathbf{N}_{\mathbf{t}}$ of WFA-labeled PNNs by TOD for each group according to methods used to detect similar relationships in postmortem studies (Zhou et al., 2001; Hofman, 2003; Li et al., 2013). Quartic regression models were used as described previously (Pantazopoulos et al., 2017) to fit expression patterns reported in the mouse and human amygdala consisting of two peaks and two troughs (Albrecht et al., 2013; Pantazopoulos et al., 2017).

\section{Numerical densities (mouse samples)}

Numerical densities were calculated as $\mathbf{N}_{\mathrm{d}}=\sum \mathbf{N} / \sum \mathbf{V}$ where $\mathrm{N}=$ sum of all PNNs counted in each region for each animal, and $\mathbf{V}$ is the volume of each region per animal, calculated as $\mathbf{V}=\sum \mathbf{a} \bullet \mathbf{z}$, where $\mathbf{z}$ is the thickness of each section $(30 \mu \mathrm{m})$ and $a$ is area in $\mu \mathrm{m}^{2}$. Rhythmic relationships of PNNs and cathepsin-S in mice were analyzed by plotting means and SD per each time point across the 24-h cycle, as conducted in previous studies (Lamont et al., 2005; Segall et al., 2009; Harbour et al., 2014).

\section{Numerical densities and total numbers estimates (human samples)}

Total number $\left(\mathbf{N}_{\mathrm{t}}\right)$ of WFA-labeled PNNs was calculated as $\mathbf{N}_{\mathbf{t}}=\mathbf{i} \bullet \mathbf{\Sigma} \mathbf{n}$, where $\Sigma n=$ sum of the cells counted in each subject, and $\mathbf{i}$ is the section interval (i.e., number of serial sections between each section and the next within each compartment $=26$ ) as described previously (Berretta et al., 2007). Numerical densities were calculated as $\mathbf{N}_{\mathbf{d}}=$ $\sum \mathbf{N} / \sum \mathbf{V}$, where $\mathbf{V}$ is the volume of each amygdala nucleus or the TRN, calculated as $\mathbf{V}=\mathbf{z} \bullet \mathbf{s s f} \bullet \mathbf{\Sigma} \mathbf{a}$, where $\boldsymbol{z}$ is the thickness of the section $(40 \mu \mathrm{m})$, ssf is the section sampling fraction $(1 / 26$; i.e., number of serial sections between each section within a compartment), and $\mathbf{a}$ is the area of the region of interest.

\section{Animals}

Adult male wild-type C57/BL6 mice housed in individual wheel-running cages in a 12/12 h light/dark (LD) cycle were used to examine diurnal rhythms of PNN composition. Three male C57/BL6 mice were killed every $4 \mathrm{~h}$ across the 24-h cycle at ZTO, ZT4, ZT8, ZT12, ZT16, and ZT20. A separate set of adult male C57/BL6 mice were used to test for circadian rhythms of PNN composition. Mice were housed in a 12/12 LD cycles for four weeks, followed by three full 24-h cycles in constant darkness, then killed every $4 \mathrm{~h}$ at circadian time (CT)0, CT4, CT8, CT12, CT16, and CT20, three mice per time point. Wheel-running actigraphs were used to determine individual CT times for killing animals housed in constant darkness. Activity onset over three 24-h cycles was used to predict CT time in the fourth cycle during which animals were killed. All animals in the constant darkness study were killed under dim red light conditions. Circadian rhythm of each mouse was monitored with ClockLab (Actimetrics) using wheel-running activity data. Mice were killed using cervical dislocation in the light or in the dark using a dim red light, depending on lighting conditions at time of killing. Mice were perfused intracardially with $4 \%$ PFA, and brains were stored in $0.1 \mathrm{M} \mathrm{PB}$ with $0.1 \% \mathrm{Na}$ azide and $30 \%$ sucrose. Brains were then sliced into serial $30-\mu \mathrm{m}$ brain sections on an American Optical freezing microtome. The housing and treatment of experimental animals were approved by the University of Mississippi Medical Center Institutional Animal Care and Use Committee and followed guidelines set by the National Institutes of Health.

\section{Human subjects and tissue processing}

Tissue blocks containing the whole amygdala or thalamus from 15 donors were obtained from the Harvard Brain Tissue Resource Center, McLean Hospital, Belmont, MA (Tables 1 and 2). Diagnoses were made by two psychiatrists on the basis of retrospective review of medical records and extensive questionnaires concerning social and medical history provided by family members. A neuropathologist examined several regions from each brain for a neuropathology report. The cohort for this study did not include subjects with evidence for gross and/or macroscopic brain changes, or clinical history consistent with cerebrovascular accident or other neurologic disorders. Subjects with Braak and Braak Stages III or higher were not included. Subjects had no significant history of psychiatric illness, or substance dependence, other than nicotine and alcohol, within 10 years from death. 
Table 1: TRN sample demographic and descriptive characteristics

\begin{tabular}{|c|c|c|c|c|c|c|c|}
\hline Case & Age & Sex & Cause of death & Brain weight (g) & PMI (hrs) & Hemisphere & TOD \\
\hline S05735 & 74 & $\mathrm{~F}$ & Cancer (C) & 1145 & 12.2 & $\mathrm{~L}$ & 14.00 \\
\hline S13845 & 37 & $M$ & Electrocution $(\mathrm{A})$ & 1460 & 18.75 & $\mathrm{R}$ & 21.00 \\
\hline S10160 & 85 & $M$ & Cancer (C) & 1225 & 20.3 & L & 05.30 \\
\hline S18228 & 78 & $\mathrm{~F}$ & Cancer (C) & 1100 & 23.9 & L & 05.00 \\
\hline S07594 & 95 & $\mathrm{~F}$ & Unknown & 1350 & 7.1 & $\mathrm{R}$ & 14.50 \\
\hline S07429 & 68 & $\mathrm{~F}$ & Unknown & 1230 & 24.8 & $\mathrm{R}$ & 19.45 \\
\hline S14342 & 70 & $\mathrm{~F}$ & Cardiac arrest $(\mathrm{A})$ & 1245 & 18.0 & $\mathrm{R}$ & 07.29 \\
\hline S08987 & 53 & $\mathrm{~F}$ & Cancer (C) & 1330 & 24.0 & $\mathrm{R}$ & 08.32 \\
\hline S11774 & 74 & $M$ & Cardiac arrest $(\mathrm{A})$ & 1490 & 15.81 & $\mathrm{R}$ & 08.41 \\
\hline S03774 & 70 & $M$ & Aortic aneurysm (A) & 1400 & 17.3 & $\mathrm{R}$ & 20.46 \\
\hline S17165 & 58 & $\mathrm{~F}$ & COPD (C) & 1345 & 17.8 & $\mathrm{R}$ & 00.35 \\
\hline
\end{tabular}

Tissue blocks were dissected from fresh brains and postfixed in $0.1 \mathrm{M}$ PB containing $4 \%$ paraformaldehyde and $0.1 \mathrm{M} \mathrm{Na}$ azide at $4{ }^{\circ} \mathrm{C}$ for three weeks, cryoprotected at $4^{\circ} \mathrm{C}$ for three weeks (30\% glycerol, $30 \%$ ethylene gly$\mathrm{col}$, and $0.1 \% \mathrm{Na}$ azide in $0.1 \mathrm{M} \mathrm{PB}$ ), embedded in agar, and presliced in 2-mm coronal slabs using an Antithetic Tissue Slicer (Stereological Research Lab.). Each slab was exhaustively sectioned using a freezing microtome (American Optical 860). Sections were stored in cryoprotectant at $-20^{\circ} \mathrm{C}$. Using systematic random sampling criteria, sections through the amygdala were serially distributed in 26 compartments (40- $\mu$ m-thick sections; 610 sections/compartment; $1.04-\mathrm{mm}$ section separation within each compartment). All sections within one compartment/subject were selected for histochemistry (i.e., WFA), thus respecting the "equal opportunity" rule (Coggeshall and Lekan, 1996; Gundersen et al., 1999).

\section{Sleep deprivation}

Adult male wild-type C57/Bl6 mice housed in 12/12 LD cycle were used for sleep deprivation experiments. Mice were either sleep deprived using gentle handling for $5 \mathrm{~h}$ from lights on (7 A.M.) to 12 P.M. $(n=12)$ or handled during the dark phase for $5 \mathrm{~h}$ the night before (controls; $n=12$ ), to control for potential confounding effects of handling on the outcome measures. Mice were killed immediately following $5 \mathrm{~h}$ of sleep deprivation, and control mice were killed at the same time (ZT5: 12 P.M.). Mice were perfused intracardially with $4 \%$ PFA, and brains were stored in $0.1 \mathrm{M} \mathrm{PB}$ with $0.1 \% \mathrm{Na}$ azide and $30 \%$ sucrose. Brains were then sliced into serial $30-\mu \mathrm{m}$ brain sections on an American Optical freezing microtome. WFA labeling was used to quantify PNNs in the habenula, prefrontal cortex, amygdala, thalamus, and hippocampus using stereology-based sampling methods.

\section{Mouse auditory fear conditioning}

Auditory contextual fear conditioning was conducted as described previously (Gisabella et al., 2016). Mice were placed in a fear conditioning box at ZTO (7 A.M.; $64 \mathrm{~cm}$ wide, $73 \mathrm{~cm}$ deep, and $68 \mathrm{~cm}$ high; Med Associates) placed in a larger, sound-attenuating chamber. Precisely four mice will be placed in four boxes chamber (one mouse for each box) at the same time for experimental

Table 2: Amygdala sample demographic and descriptive characteristics

\begin{tabular}{|c|c|c|c|c|c|c|c|}
\hline Case & Age & Sex & Cause of death & Brain weight (g) & PMI (hrs) & Hemisphere & $\overline{\text { TOD }}$ \\
\hline$\overline{S 90122}$ & 70 & $\mathrm{M}$ & Cardiac arrest $(\mathrm{A})$ & 1360 & 23.2 & $\mathrm{~L}$ & 12.17 \\
\hline S12827 & 71 & $\mathrm{M}$ & Cardiac arrest $(\mathrm{A})$ & 1580 & 24.0 & $\bar{L}$ & 10.10 \\
\hline S07340 & 65 & $M$ & Cardiac arrest $(\mathrm{A})$ & 1240 & 17.3 & $\mathrm{~L}$ & 06.45 \\
\hline S08987 & 53 & $\mathrm{~F}$ & Cancer (C) & 1330 & 24.0 & $\mathrm{R}$ & 08.32 \\
\hline S30877 & 62 & $\mathrm{M}$ & Cardiac arrest $(\mathrm{A})$ & 1300 & 29.2 & $\mathrm{~L}$ & 21.18 \\
\hline S14247 & 72 & $\mathrm{M}$ & Cardiac arrest $(\mathrm{A})$ & 1560 & 28.2 & $\mathrm{R}$ & 07.35 \\
\hline S05735 & 74 & $\mathrm{~F}$ & Cancer (C) & 1145 & 12.2 & $\mathrm{~L}$ & 14.00 \\
\hline S16022 & 68 & $\mathrm{~F}$ & Cardiac arrest $(\mathrm{A})$ & 1330 & 14.75 & $\mathrm{R}$ & 09.30 \\
\hline S10160 & 85 & $\mathrm{M}$ & Cancer (C) & 1225 & 20.3 & $\mathrm{~L}$ & 05.30 \\
\hline S18228 & 78 & $\mathrm{~F}$ & Cancer (C) & 1100 & 23.9 & $\mathrm{~L}$ & 05.00 \\
\hline Total $/$ mean $\pm \mathrm{SD}$ & $65.4 \pm 12.2$ & $5 F 9 M$ & & $1315.0 \pm 161.3$ & $21.8 \pm 5.7$ & $8 \mathrm{~L} 6 \mathrm{R}$ & \\
\hline
\end{tabular}

A, acute death, no prolonged agonal period; C, chronic, prolonged agonal period; COPD, chronic obstructive pulmonary disease; PMI, postmortem time interval. 
A

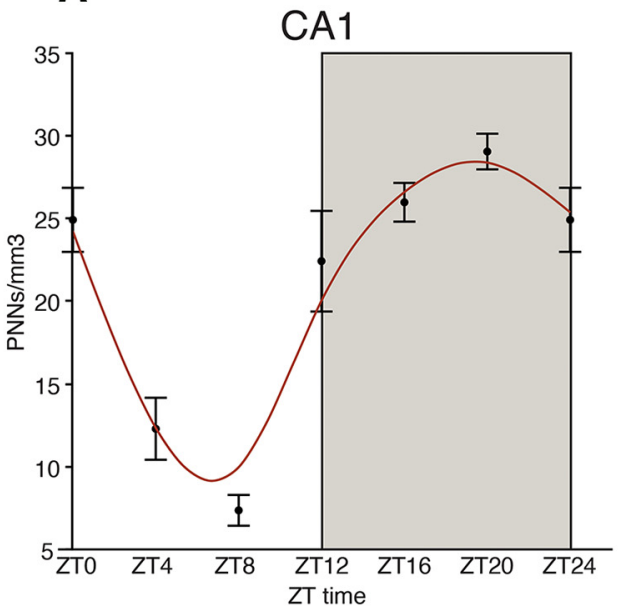

C

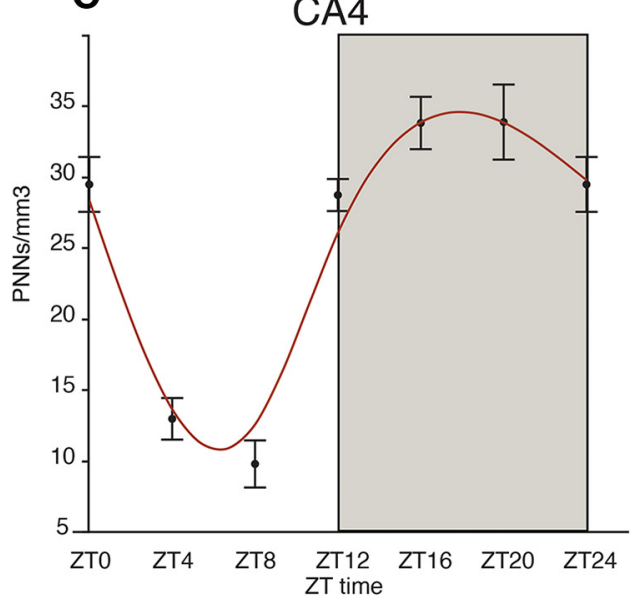

E

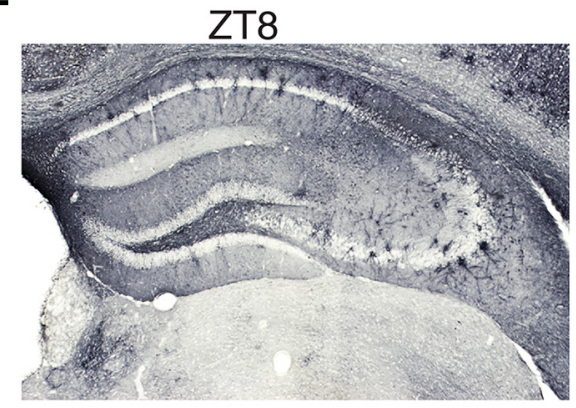

B
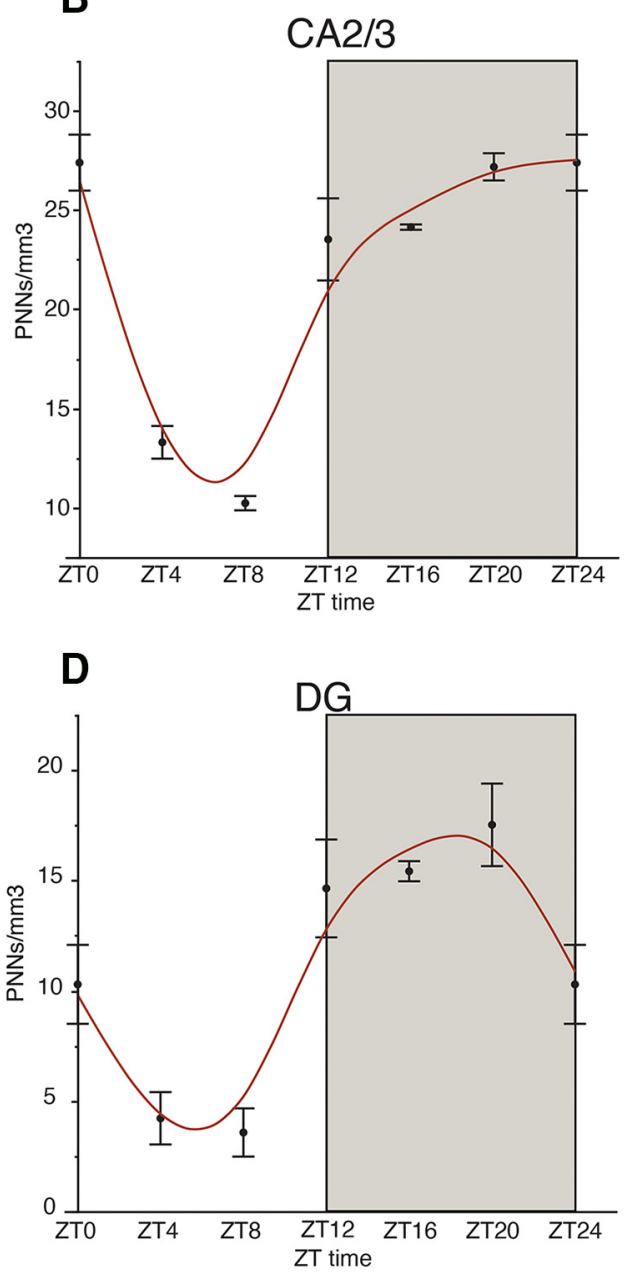

$\mathbf{F}$

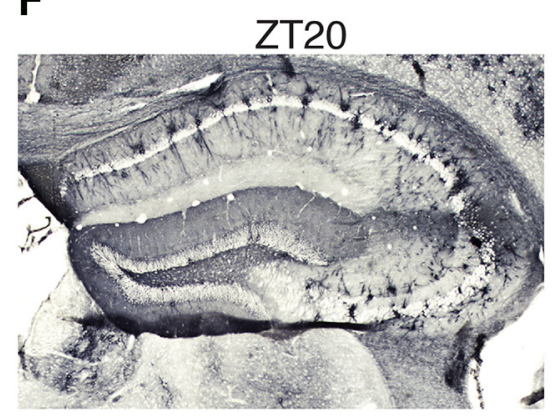

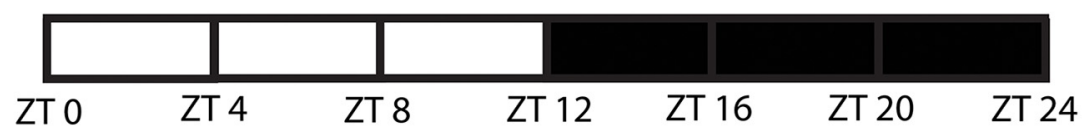

Figure 1. Diurnal rhythms of PNNs in the mouse hippocampus. Analysis of WFA+ PNNs across the 24-h cycle in male mice housed in a 12/12 h LD cycle revealed a diurnal rhythm of WFA+ PNNs in hippocampal sectors CA1 (A) CA2/3 (B), CA4 (C), and the DG $(\boldsymbol{D})$ with peaks at $\sim$ ZT20 and troughs at $\sim$ ZT8. Error bars represent SD. Representative low-magnification images of WFA labeling in the mouse hippocampus at ZT8 $(\boldsymbol{E})$ and ZT20 $(\boldsymbol{F})$.

comparison. Mice remained in the chamber for 3 min before delivery of four tones, each of 10 -s duration $(85 \mathrm{~dB}$, $10 \mathrm{kHz}$ ). Each tone was followed by a footshock lasting $2 \mathrm{~s}$ (0.8-mA amplitude) pairings were administered (60- to 200 -s variable inter tone interval; total time mice spent inside the chamber was 15-18 min). After the test, the mice were placed back into normal housing (four control mice, or sleep deprived for $5 \mathrm{~h}$ before being placed back into normal housing (four mice).

Mice were returned to the context on the second day for an extinction session (10-min total inside the chamber with no shock and tone) then placed back in their cages. 

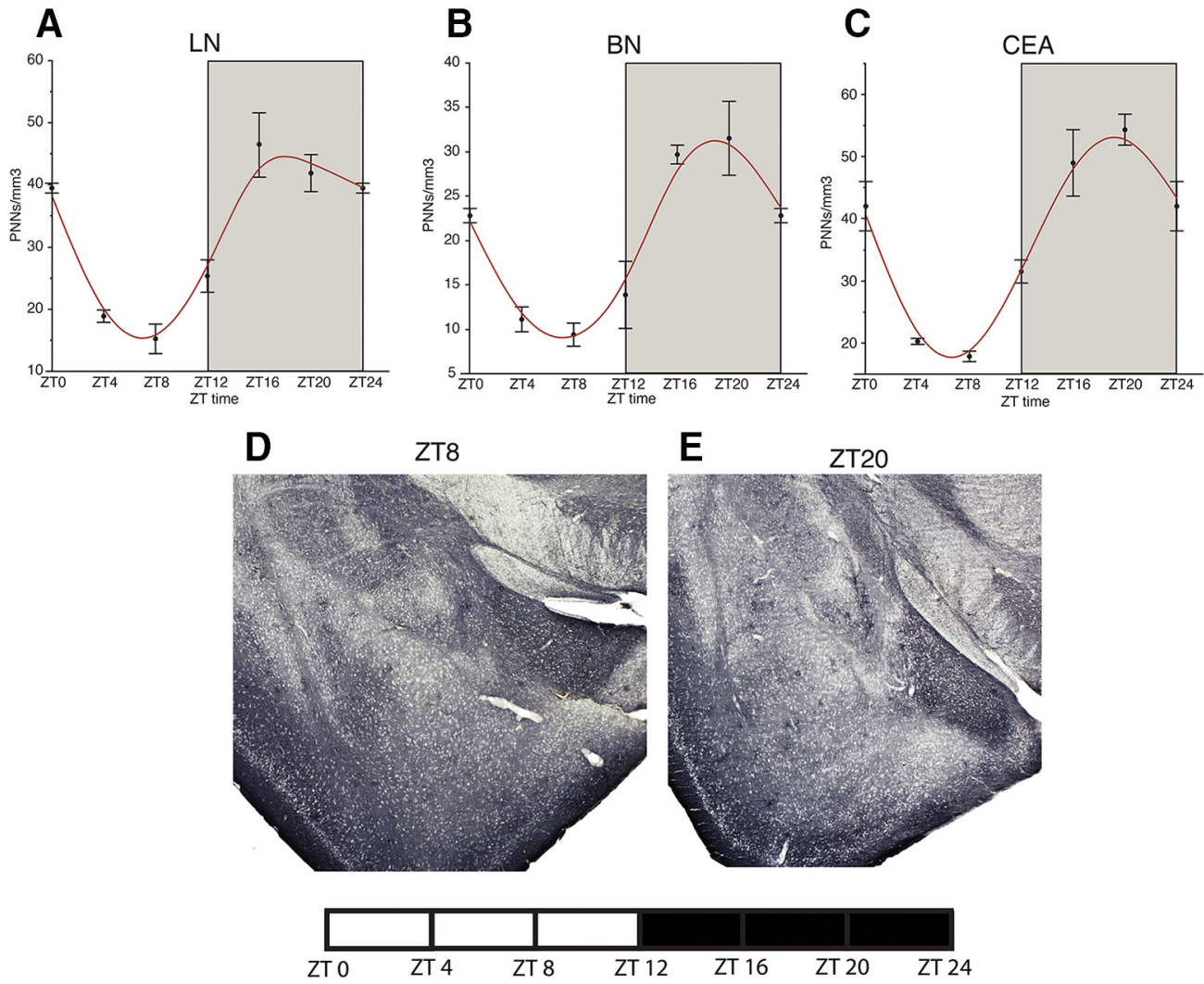

Figure 2. Diurnal rhythms of PNNs in the mouse amygdala. Diurnal rhythms of WFA+ PNNs were observed in the lateral amygdala $(\boldsymbol{A})$ basal amygdala $(\boldsymbol{B})$, and central amygdala $(\boldsymbol{C})$ with peaks at $\sim$ ZT20 and troughs at $\sim$ ZT8. Error bars represent SD. Representative low-magnification images of WFA labeling in the mouse amygdala at ZT8 (D) and ZT20 (E).

Mice were placed in a novel context on the third day at 7 A.M. for auditory fear extinction inside the chamber for a total time of $5 \mathrm{~min}(3 \mathrm{~min}$ pretone; $60 \mathrm{~s}, 85 \mathrm{~dB}$, $10 \mathrm{kHz}$ tone; $60 \mathrm{~s}$ posttone period). Low freezing before the onset of tone presentation indicated that animals did not generalize fear to the novel context, and also enabled us to conclude that freezing observed during the tone was evoked specifically by the tone. Freezing behavior was defined as periods of at least $1 \mathrm{~s}$ with the complete absence of movement except breathing; it was measured with manual scoring. The percent of time spent freezing during intervals of interest was quantified, and these results were analyzed using ANOVA. Post hoc Fisher's PLSD tests were performed after a significant omnibus $F$ ratio.

\section{Cathepsin-S PNN digestion}

Free floating mouse brain sections were incubated with $300 \mathrm{ng}$ of active human cathepsin-S (SRP0292, Sigma-Aldrich), in activation buffer containing $1.8 \mathrm{~mm}$ DTT, $1.8 \mathrm{~mm}$ EDTA, 1\% BSA, $12 \mathrm{~mm}$ citric acid, and 43 mм $\mathrm{Na}_{2} \mathrm{HPO}_{4}$ at $37^{\circ} \mathrm{C}$ for either $3 \mathrm{~h}$ or $24 \mathrm{~h}$. Control sections were incubated in activation buffer (1.8 mM DTT, $1.8 \mathrm{~mm}$ EDTA, 1\% BSA, $12 \mathrm{~mm}$ citric acid, and $43 \mathrm{~mm}$ $\mathrm{Na}_{2} \mathrm{HPO}_{4}$ ) at $37^{\circ} \mathrm{C}$ in parallel. Following cathepsin-S incubation, sections were labeled with WFA and WFA+ PNNs were quantified in the hippocampus as described above.

\section{Results}

We use the chronobiology term "circadian" to refer to rhythms observed in constant darkness, regulated by endogenous circadian processes in the absence of environmental signals that can entrain rhythms such as light-dark cycles. We use the term "diurnal" to refer to rhythms observed in light-dark cycles, which may reflect immediate responses to environmental cycles rather than true circadian rhythms.

\section{Diurnal rhythms of PNNs in the mouse brain}

In a cohort of adult male C57/BL6 mice, housed in a 12/ 12 LD cycle, we observed diurnal rhythms in the density of WFA + PNNs in the hippocampus, amygdala, prefrontal cortex, habenula, and TRN (Figs. 1-5). WFA+ PNN rhythms in the hippocampal sectors displayed consistent peaks at ZT20, and troughs at ZT6 across hippocampal sectors (Fig. 1). WFA+ PNN density rhythms in the amygdala were similar to hippocampal rhythms, with peaks at ZT20 and troughs at ZT8 across amygdala nuclei (Fig. 2). Similar relationships were observed in the prefrontal cortex, with WFA+ PNN densities displaying peaks at approximately ZTO and troughs at ZT8 (Fig. 3). WFA+ PNN density rhythms in the habenula (Fig. 4) and TRN (Fig. 5) also displayed consistent diurnal rhythms, with peaks at approximately ZT20 and troughs at ZT8. ANCOVA models testing the main effect of $Z T$ time and the effect of average daily amount of wheel running activity showed significant effects of ZT time on WFA+ PNN densities in all regions 

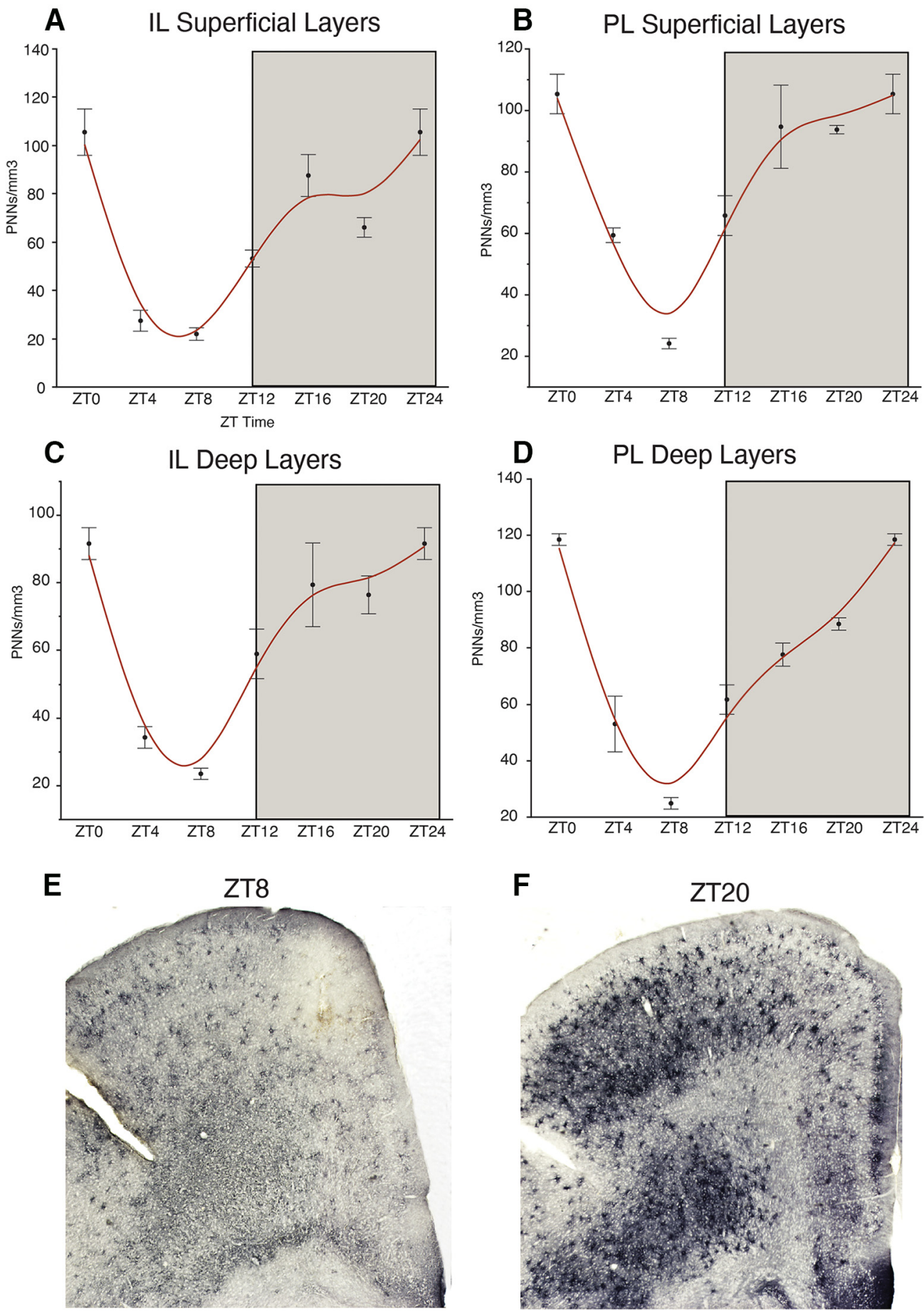

$\mathbf{F}$
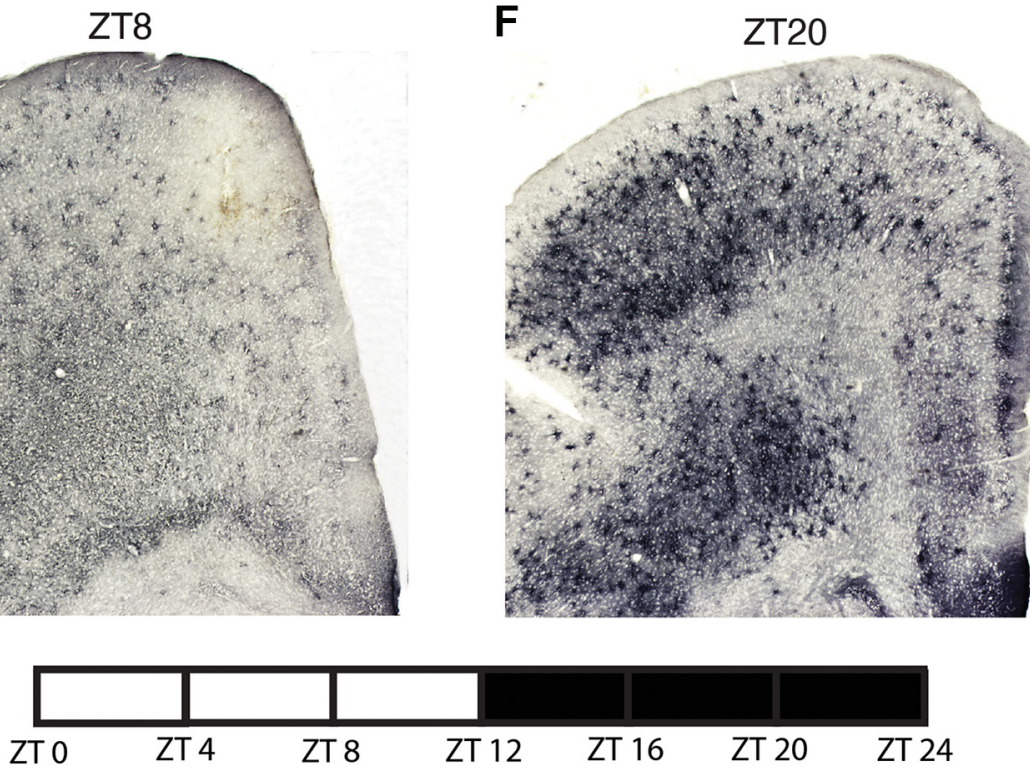

Figure 3. Diurnal rhythms of PNNs in the mouse prefrontal cortex. Diurnal rhythms of WFA+ PNNs were observed in the infralimbic superficial $(\boldsymbol{A})$ prelimbic superficial $(\boldsymbol{B})$, infralimbic deep $(\boldsymbol{C})$, and prelimbic deep $(\boldsymbol{D})$ layers of the mouse, with peaks at $\sim$ ZT0 and troughs at $\sim$ ZT8. Error bars represent SD. Representative low-magnification images of WFA labeling in the mouse prefrontal cortex at ZT8 $(\boldsymbol{E})$ and ZT20 $(\boldsymbol{F})$. 

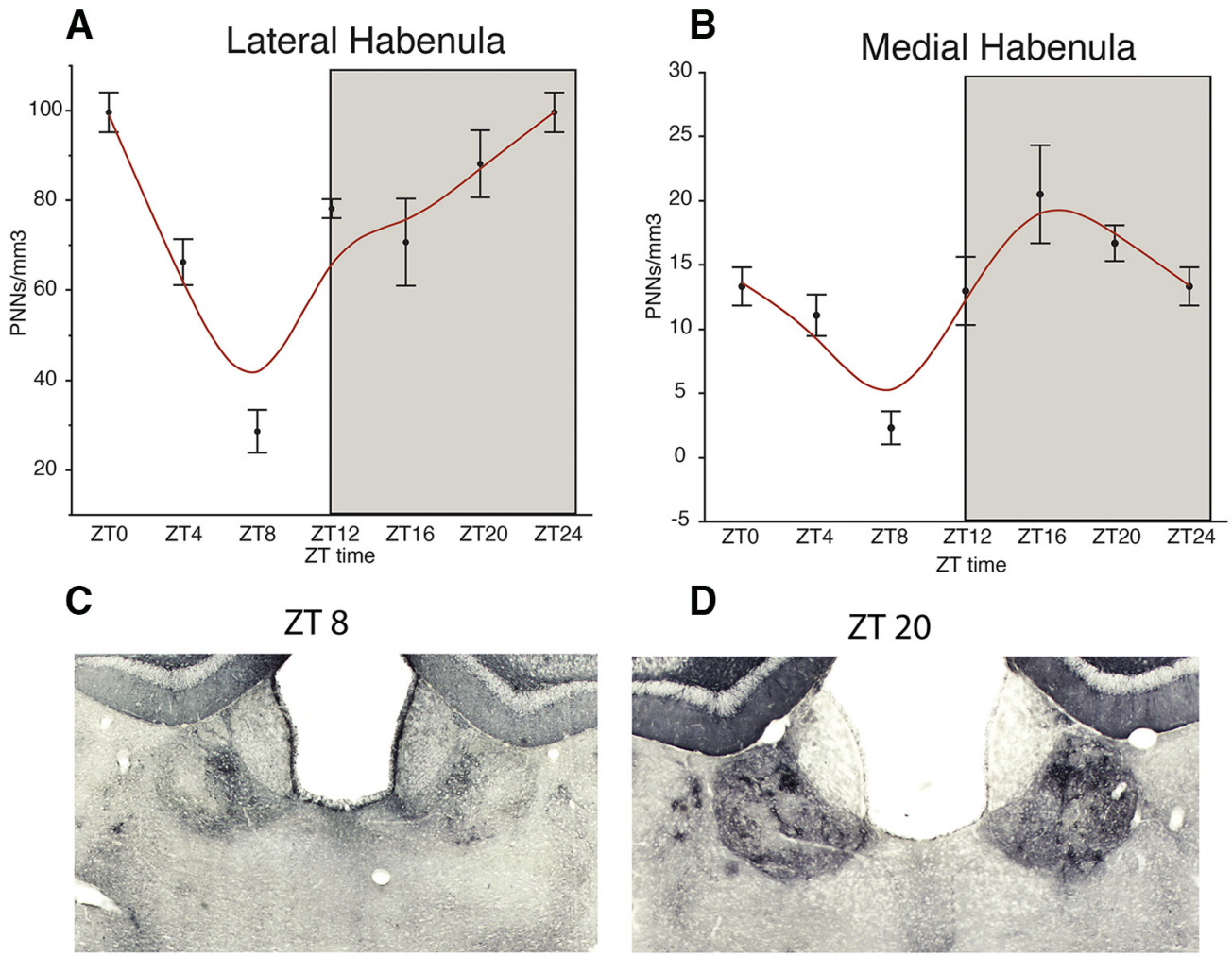

D
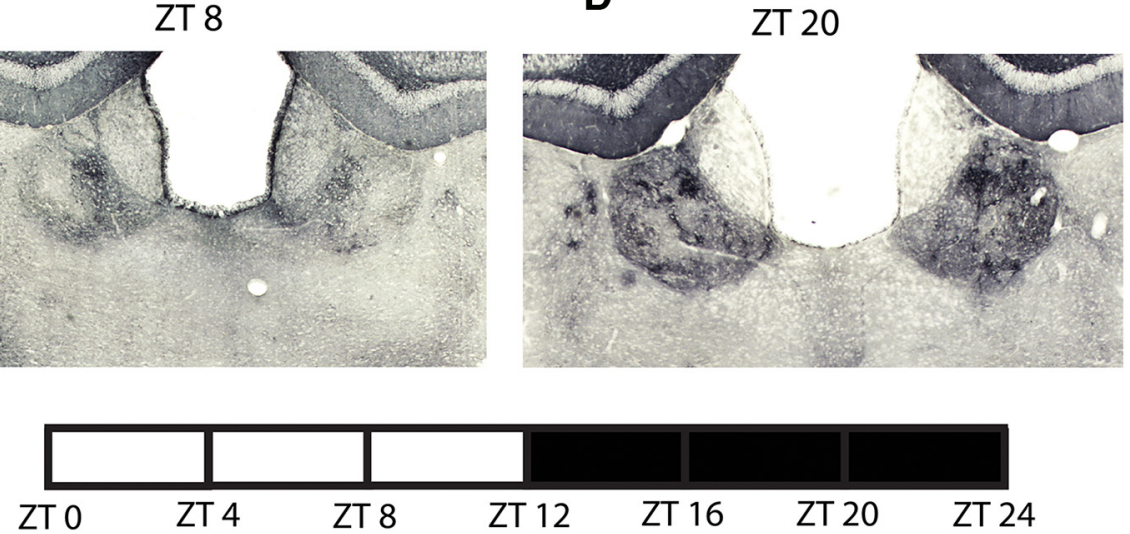

Figure 4. Diurnal rhythms of PNNs in the mouse habenula. Analysis of WFA+ PNNs across the 24-h cycle in male mice housed in a $12 / 12 \mathrm{~h}$ LD cycle revealed a diurnal rhythm of WFA+ PNNs in the lateral habenula $(\boldsymbol{A})$ and medial habenula $(\boldsymbol{B})$, with a peak at $\sim$ ZT0 and trough at $\sim$ ZT8 for the lateral habenula, and a peak at $\sim$ ZT16 and trough at $\sim$ ZT8 for the medial habenula. Error bars represent SD. Representative low-magnification images of WFA labeling in the mouse habenula at ZT8 (C) and ZT20 (D).

examined (Table 3). In comparison, average daily amount of wheel-running activity showed a significant effect on densities of WFA + PNNs in only the central amygdala and TRN (Table 3).

\section{Circadian rhythms of PNNs in the mouse brain}

These studies were designed to assess whether diurnal rhythms in mice reflect a true circadian rhythm, and to confirm the existence of PNN density rhythms in a separate strain of mice. We used adult male C57/BI6 mice housed in a 12/12 LD cycle and then placed into constant darkness for three full 24-h cycles to quantify WFA+ PNN rhythms in free-running circadian conditions. In mice kept in constant darkness, numerical density of WFA+ PNNs displayed circadian rhythms in all regions identical to diurnal rhythms described above, with consistent peaks at approximately CT20 and troughs at approximately CT8 across regions (Fig. 6).

\section{Sleep deprivation prevents the decrease of PNNs during the day in the mouse hippocampus}

Sleep deprivation by gentle handling has been previously shown to prevent synaptic modification that occurs in the hippocampus during sleep in rodents (Havekes et al., 2016; Raven et al., 2019). Here, we use the same approach to test the hypothesis that sleep deprivation prevents the decrease of WFA+ PNN densities in the mouse hippocampus. Mice that were sleep deprived for $5 \mathrm{~h}$ (ZT0-ZT5) starting from the beginning of the light cycle had significantly higher numerical density of WFA + PNNs in the dentate gyrus $(p=0.01)$ and sectors CA4 $(p=0.01)$, CA3/2 $(p=0.01)$, and CA1 $(p=0.001$; Fig. $7 B-D)$. Similar differences in WFA+ PNN densities were observed in the amygdala, habenula, and prefrontal cortex (Fig. 7E-G). In a set of animals that underwent auditory fear conditioning, $5 \mathrm{~h}$ of sleep deprivation significantly enhanced fear memory extinction (Fig. 7A).

\section{Association of cathepsin-S microglia with diurnal PNN rhythms}

Cathepsin-S has been reported to be rhythmically expressed in the mouse prefrontal cortex and associated with diurnal rhythms in dendritic spines and electrophysiological properties of prefrontal cortex neurons (Hayashi et al., 2013b). As a first step in testing whether cathepsin$\mathrm{S}$ may contribute to circadian modification of PNN integrity, we tested the hypothesis that its expression in microglia may vary according to a diurnal rhythm, antiphase to 

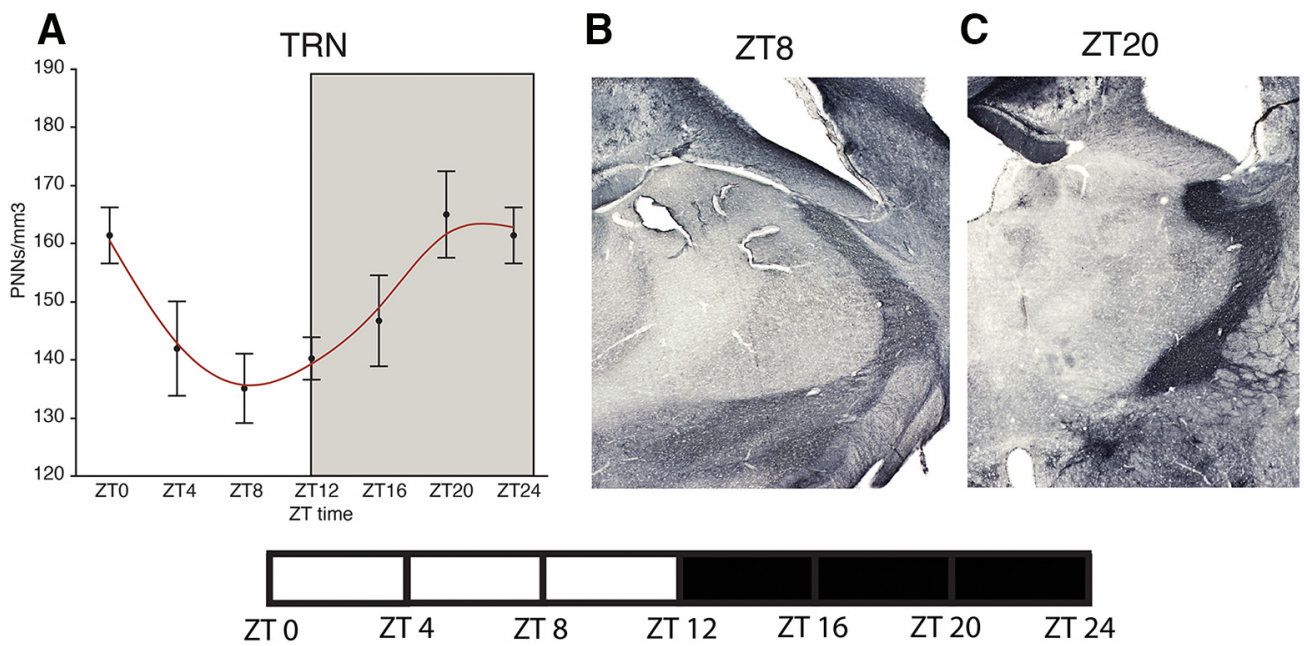

Figure 5. Diurnal rhythms of PNNs in the mouse TRN. Analysis of WFA+ PNNs across the 24-h cycle in male mice housed in a 12/ $12 \mathrm{~h}$ LD cycle revealed a diurnal rhythm of WFA+ PNNs in the TRN $(\boldsymbol{A})$ with a peak at $\sim$ ZT20 and a trough at $\sim$ ZT8. Error bars represent SD. Representative low-magnification images of WFA labeling in the mouse TRN at ZT8 (B) and ZT20 (C).

WFA+ PNN rhythms. We observed a diurnal rhythms of cathepsin-S-IR microglia densities in the mouse hippocampus, antiphase to the rhythms of WFA+ PNNs in this region (Fig. 8), with peaks at approximately ZT6 and troughs at ZTO. Similar diurnal cathepsin-S rhythms were observed in the amygdala and prefrontal cortex (Fig. 9). No significant effects of average daily wheel-running activity on cathepsin-S-IR cell densities were observed (Table 3). Finally, to confirm that cathepsin-S degrades
PNNs, we incubated mouse sections in active cathepsin-S ( 3 and 24 h). Our results show an incubation time-dependent decrease of WFA+ PNN labeling, with a significant $54.5 \%$ decrease after $3 h(p<0.02)$ and a complete elimination after $24 \mathrm{~h}(p<0.0001$; Fig. 10A- $)$. Dual immunohistochemistry confirmed that virtually all (88.12-92.95\%) of cathepsin-S-IR cells in the mouse hippocampus, infralimbic and prelimbic cortex, amygdala, habenula, and TRN correspond to IBA1-positive microglia (Fig. 10G-N).

Table 3: Summary table of ZT time and average daily running activity effects on WFA + PNN and CatS-IR cell densities

\begin{tabular}{|c|c|c|c|c|}
\hline & $\begin{array}{l}\mathrm{ZT} \text { time } \\
F \text { ratio }\end{array}$ & $\begin{array}{l}\text { ZT time } \\
p \text { value }\end{array}$ & $\begin{array}{l}\text { Avg daily wheel running } \\
\text { activity } F \text { ratio }\end{array}$ & $\begin{array}{l}\text { Avg daily wheel running } \\
\text { activity } p \text { value }\end{array}$ \\
\hline CA2/3 PNNs & 20.21 & $<0.0001$ & 4.07 & 0.07 \\
\hline DG PNNs & 7.38 & 0.003 & 0.02 & 0.91 \\
\hline Lateral amygdala PNNs & 9.16 & 0.001 & 0.02 & 0.98 \\
\hline Basolateral amygdala PNNs & 10.81 & 0.0007 & 1.72 & 0.22 \\
\hline Lateral habenula PNNs & 10.25 & 0.0009 & 0.88 & 0.37 \\
\hline Medial habenula PNNs & 5.31 & 0.01 & 0.07 & 0.78 \\
\hline IL superficial PNNs & 14.72 & 0.001 & 0.03 & 0.96 \\
\hline IL deep PNNs & 10.94 & 0.003 & 0.88 & 0.38 \\
\hline PL superficial PNNs & 28.16 & 0.0002 & 2.51 & 0.16 \\
\hline PL deep PNNs & 31.77 & $<0.001$ & 0.94 & 0.37 \\
\hline Lateral amygdala CatS & 6.01 & 0.02 & 0.22 & 0.65 \\
\hline Basolateral amygdala CatS & 26.25 & 0.0002 & 3.95 & 0.09 \\
\hline Central amygdala CatS & 14.21 & 0.001 & 0.02 & 0.88 \\
\hline IL superficial CatS & 8.20 & 0.05 & 0.17 & 0.71 \\
\hline IL deep CatS & 7.67 & 0.04 & 0.50 & 0.52 \\
\hline PL superficial CatS & 28.21 & 0.009 & 0.18 & 0.70 \\
\hline PL deep CatS & 93.77 & 0.002 & 0.009 & 0.92 \\
\hline
\end{tabular}

Values represent $F$ ratios and $p$ values derived from ANCOVA models testing effects of ZT time and average daily wheel-running activity on WFA+ PNN densities and cathepsin-S-IR cell densities. Statistically significant differences are indicated in bold $=p<0.05$. 

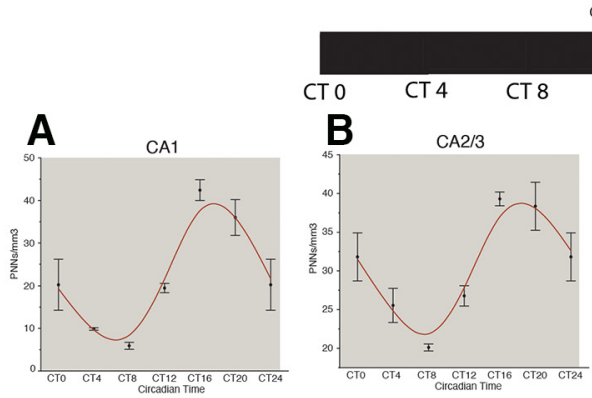

Circadian Time
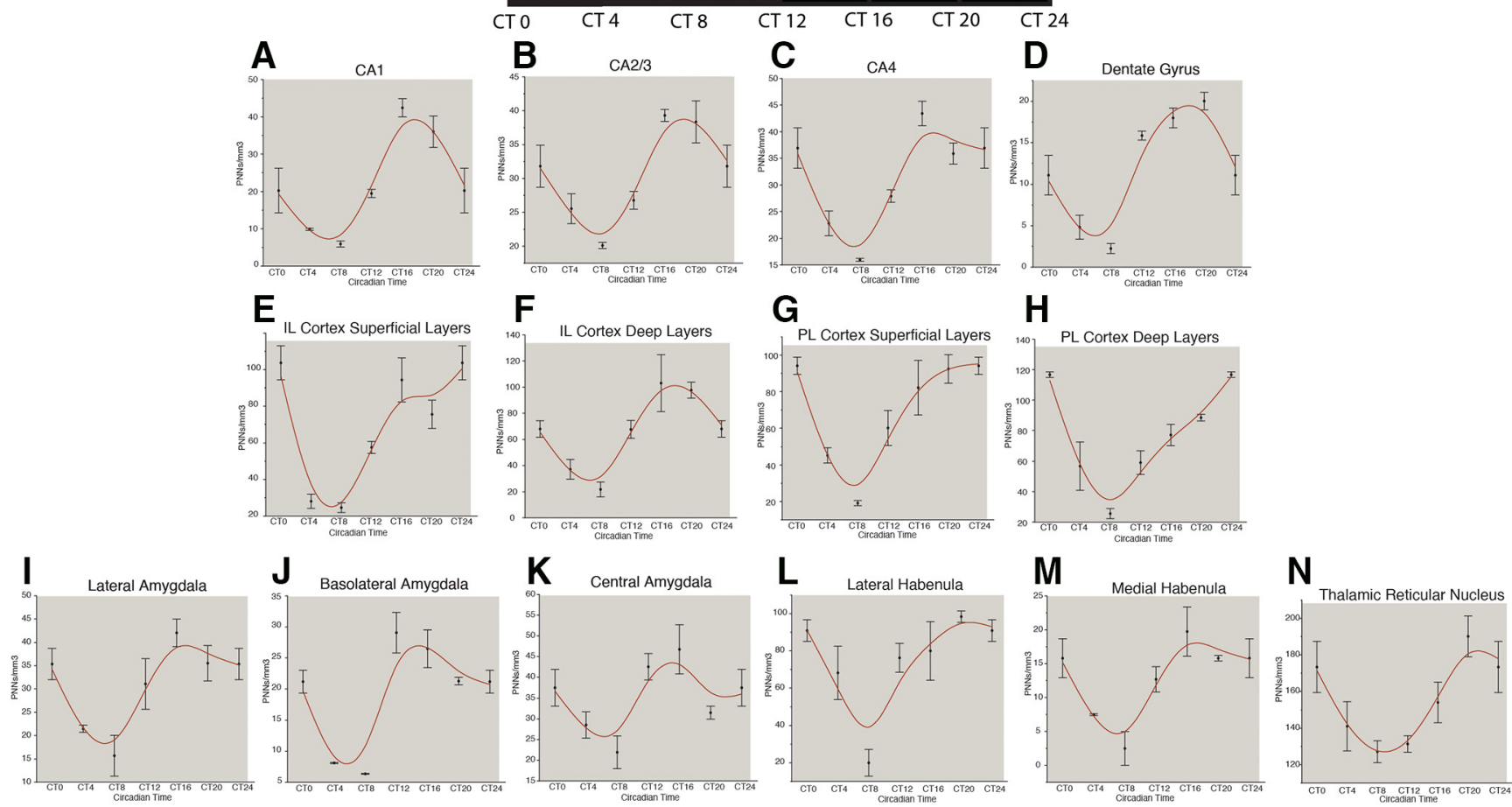

Figure 6. Circadian rhythms of PNNs in the mouse brain. Circadian rhythms in the density of WFA+ PNNs were observed in mice housed in constant darkness. In the hippocampus, these rhythms were similar to the diurnal rhythms observed in the CA regions and the DG $(\boldsymbol{A}-\boldsymbol{D})$; circadian rhythms in the density of WFA+ PNNs in the mouse prefrontal cortex also paralleled the observed diurnal rhythms in these regions, with peaks at $\sim$ CT0 and troughs at $\sim$ CT8 $(\boldsymbol{E}-\boldsymbol{H})$, with the exception of the deep layers of the IL cortex, which showed a peak at $\sim$ CT20 and trough at CT8 $(\boldsymbol{F})$. Circadian rhythms of WFA+ PNN densities were also observed in the lateral, basal, and central amygdala nuclei in constant darkness, with a peak at $\sim$ CT16 and a trough at $\sim$ CT6 (I-K). Circadian rhythms of WFA+ PNN densities in the lateral and medial habenula and TRN paralleled diurnal PNN rhythms in these regions $(\boldsymbol{L}-\mathbf{N})$. Error bars represent SDs.

\section{Diurnal rhythms of PNNs in the human amygdala and TRN}

For these studies, we used TOD for each subject as a proxy for diurnal rhythms (ZT; see Discussion) to test the hypothesis that WFA+ PNN numbers vary in a diurnal manner in the human amygdala and TRN. We observed differences in WFA+ PNN numbers in subjects with a TOD during the day in comparison to subjects with a TOD during the night in the human amygdala (Fig. 11A-D). Quartic regression analysis revealed a diurnal rhythm of WFA+ PNNs Nt in the human amygdala (Fig. 11D), with peaks at noon and midnight, and troughs at 4 A.M. and 8 P.M. In contrast, we observed day/night differences in WFA+ PNN numbers in the human TRN that are opposite to the human amygdala, with higher numbers of PNNs at night and lower numbers during the day (Fig. 11E-H). Quartic regression plots revealed peaks of WFA+ PNN numbers in the TRN at night during 4 A.M. and 8 P.M., and the lowest numbers at 12 P.M. and midnight (Fig. $11 H)$.

\section{Discussion}

We present, to our knowledge for the first time, evidence that WFA+ PNN vary according to diurnal rhythms in the human brain and to diurnal and circadian rhythms in the rodent brain. Our data add to a growing number of studies demonstrating that PNNs are dynamic structures, responding to the environment and potentially contributing to memory consolidation during sleep (Balmer et al., 2009; Brown et al., 2009; Banerjee et al., 2017; Dingess et al., 2018; Slaker et al., 2018), We show that numbers of WFA+ PNN follow diurnal rhythms in several brain regions in mouse and in human. Importantly, we show that WFA + PNN rhythmicity occurs in mice kept in constant darkness, supporting the claim that these changes reflect circadian rhythms rather than a response to light-dark cycles (Fig. 6). Our results also provide evidence for a role of the microglia-derived matrix protease cathepsin-S, known to contribute to synaptic plasticity (Hayashi et al., 2013b). We show a diurnal rhythm of cathepsin-S expression in microglia, opposite to the observed PNN rhythms, and demonstrate that cathepsin-S eliminates WFA+ PNN labeling. Taken together, these results support the hypothesis that cathepsin-S may represent one of the endogenous proteases contributing to WFA+ PNN rhythms. PNN rhythms in the mouse hippocampus coincide with reported rhythms in LTP, suggesting that WFA+ PNNs decrease during sleep, when lower levels of LTP were reported to occur (Chaudhury et al., 2005), and increase during wakefulness when higher levels of LTP 

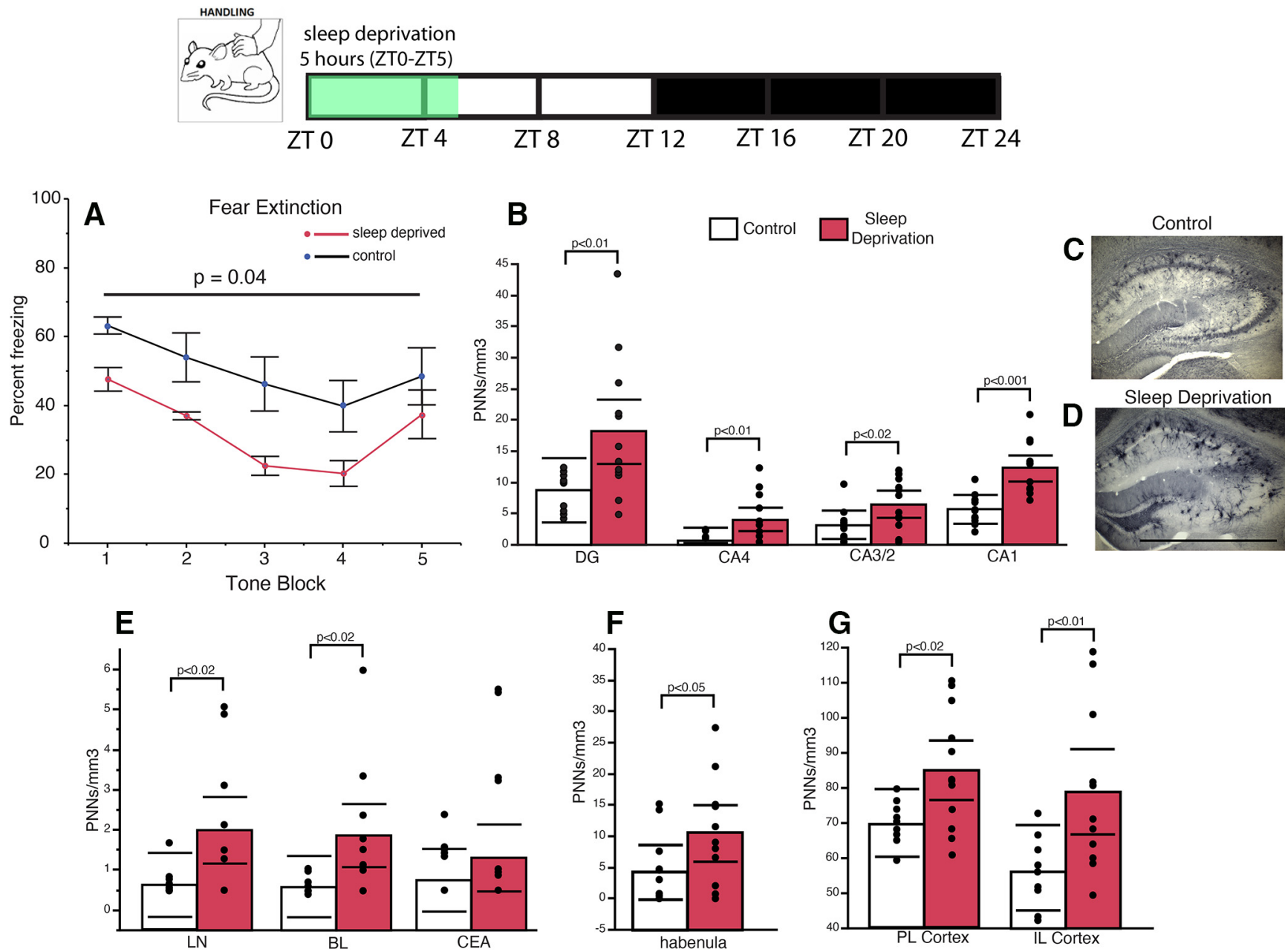

Figure 7. Sleep deprivation prevents PNN decreases. Five hours of sleep deprivation, from lights on (7 A.M.) to 12 P.M. following auditory fear conditioning, resulted in rapid extinction of fear memory $(\boldsymbol{A})$, along with significantly higher numerical density of WFA+ PNNs in the hippocampus $(\boldsymbol{B})$. Representative photomicrographs of the hippocampus labeled with WFA from a control mouse (C) and a sleep-deprived mouse $(\boldsymbol{D})$. Scale bar $=1000 \mu \mathrm{m}$. Similar increases in densities of WFA+ PNNs in SD mice were also observed in the amygdala $(\boldsymbol{E})$, habenula $(\boldsymbol{F})$, and prefrontal cortex $(\boldsymbol{G})$. Error bars represent $95 \%$ confidence intervals.

occur as animals encode new memories (Hou et al., 2013; Fig. 6). We suggest that diurnal rhythms of WFA+ PNNs in the regions examined may have broad implications for emotional memory processing and psychiatric disorders.

\section{Technical considerations}

\section{Interpretation of WFA+ PNN rhythms}

PNNs are highly complex structures formed by several glycoproteins and proteoglycans, link proteins and hyaluronan (Maeda, 2010; Miyata et al., 2012). WFA detects a specific sulfation motif on $\mathrm{N}$-acetylgalactosamine at the terminal ends of CS chains (Caterson et al., 1990; Miyata et al., 2012). CS chains can be modified by addition of sulfation groups at the two, four, or six positions along the chains, allowing for highly complex and dynamic modification (Caterson et al., 1990; Maeda, 2010; Miyata et al., 2012; Pantazopoulos et al., 2015). Furthermore, CS chains can be cleaved at varying points along the chain by several matrix proteases (Muir et al., 2002; Porter et al., 2005; Pantazopoulos et al., 2015). Together, these considerations suggest that it is unlikely that the complex PNN structure may be entirely degraded and rebuilt on a 24-h cycle. We propose that the diurnal and circadian WFA + PNN rhythms we observed may reflect modifications of the biochemical characteristics of these structures, perhaps impacting the CS chain sulfation pattern detected by WFA. It is important to emphasize that growing and compelling evidence shows that PNN and ECM functions are dictated by dynamic posttranslational modifications of their components mediated by matrix proteases (Pantazopoulos and Berretta, 2016; Lasek et al., 2018; Wen et al., 2018). Notably, these modifications determine whether effects of ECM components on synaptic plasticity are inhibitory or permissive (Miyata et al., 2012; Foscarin et al., 2017; Yang et al., 2017). Our data showing cathepsin-S rhythms antiphase to PNN rhythms, and the ability of cathepsin-S to eliminate WFA+ PNN labeling, support this interpretation and represent the first step in 
A

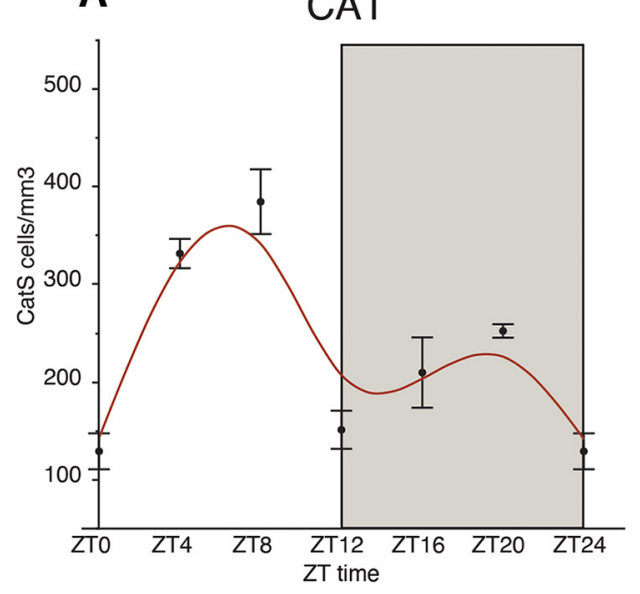

C

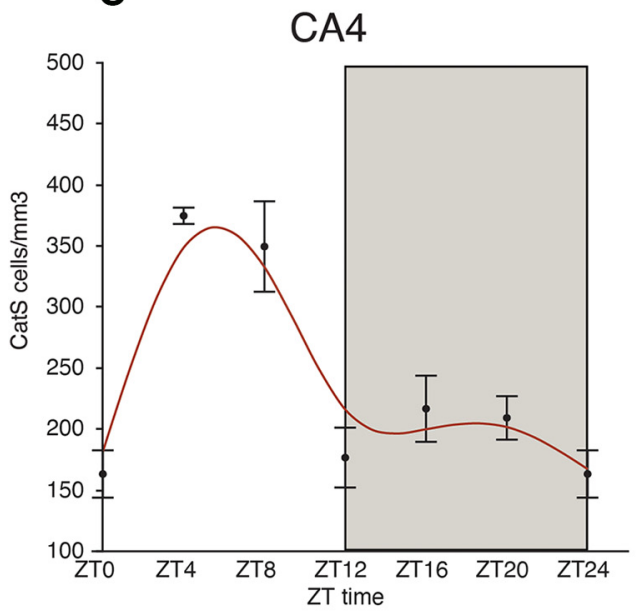

E

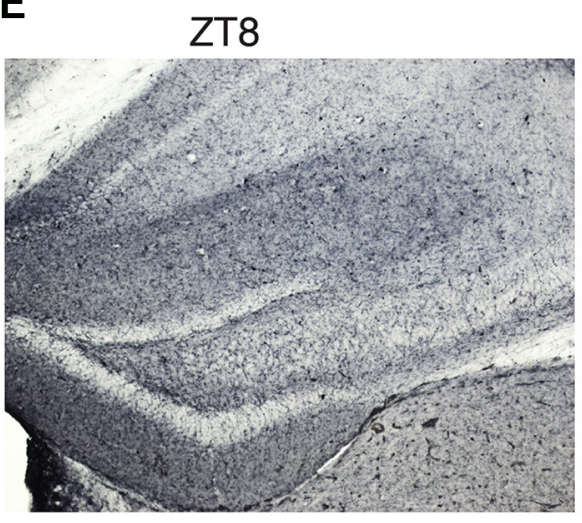

B

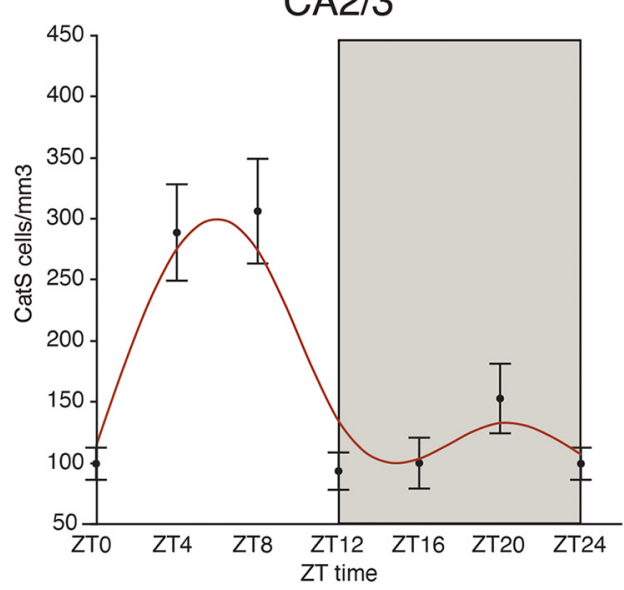

D

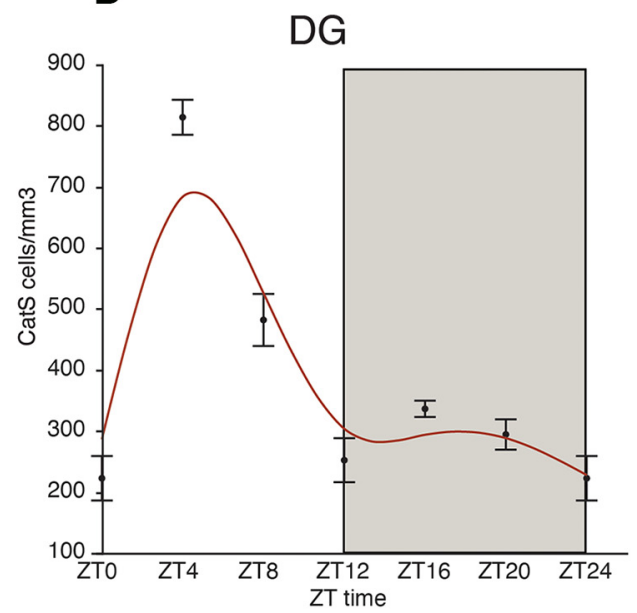

$\mathbf{F}$

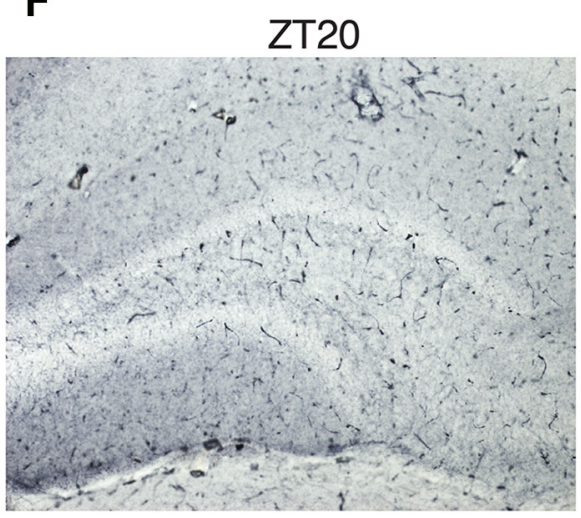

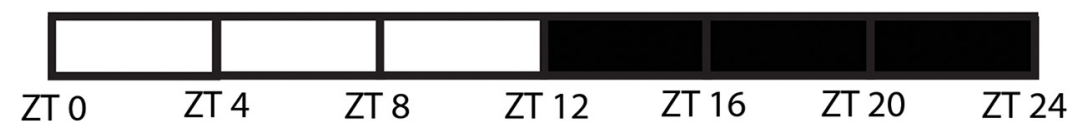

Figure 8. Cathepsin-S diurnal rhythms in the mouse hippocampus. Diurnal rhythms in densities of cathepsin-S-IR cells were observed in CA1 $(\boldsymbol{A}), \mathrm{CA} 2 / 3(\boldsymbol{B})$, CA4 $(\boldsymbol{C})$, and the DG $(\boldsymbol{D})$ in mice, with expression peaking during the middle of the light cycle, when WFA+ PNN numbers are low in these regions, and decreasing during the dark cycle, when WFA+ PNN densities are high. Error bars represent SD. Representative photomicrographs of the hippocampus labeled with cathepsin-S at ZT8 (E) and ZT20 (F). 

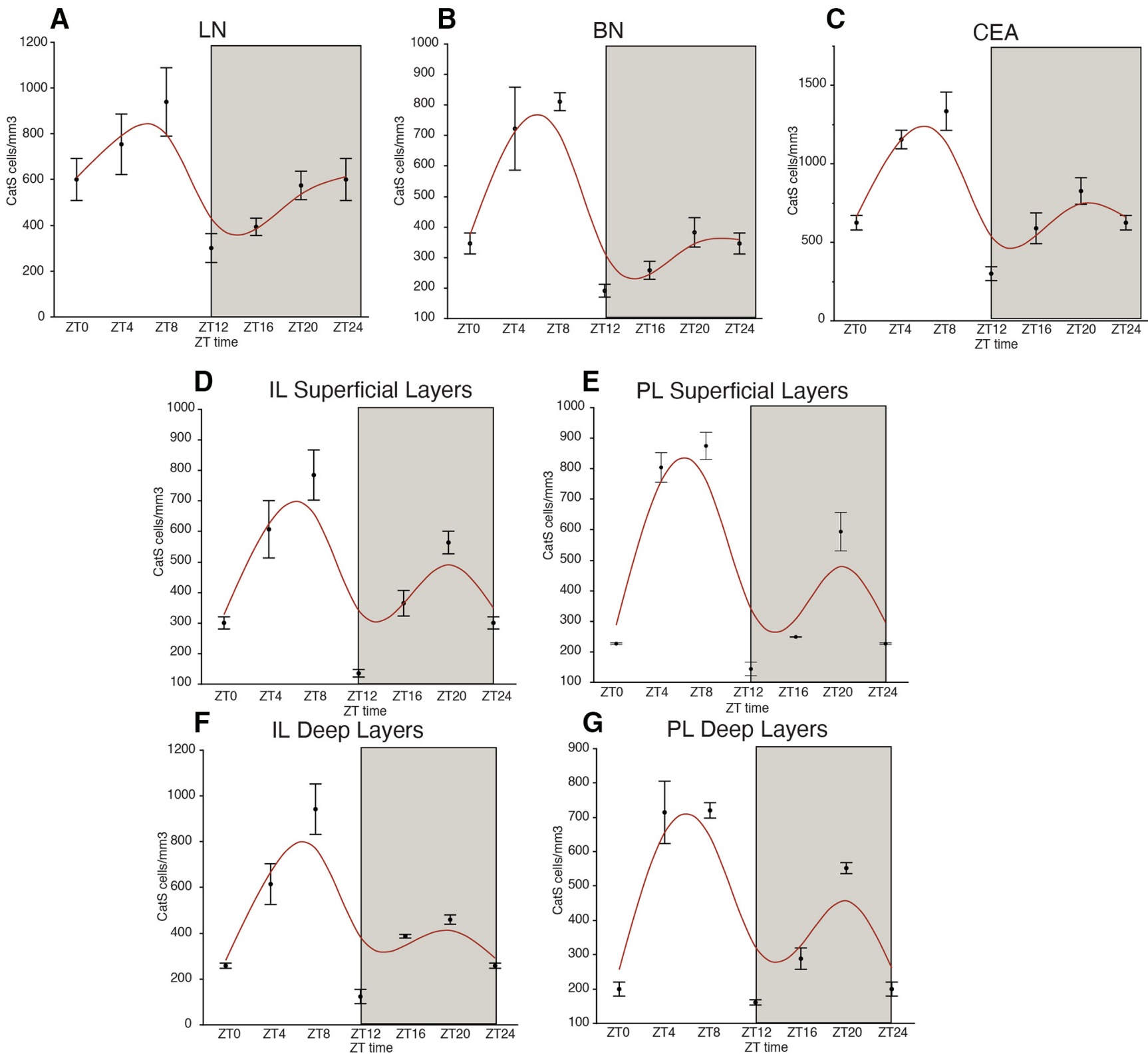

Figure 9. Cathepsin-S diurnal rhythms in the mouse amygdala and prefrontal cortex. Diurnal rhythms in densities of cathepsin-S-IR cells were observed in the lateral amygdala $(\boldsymbol{A})$, basal amygdala $(\boldsymbol{B})$, and central amygdala $(\boldsymbol{C})$, with expression peaking during the middle of the light cycle, when WFA+ PNN numbers are low in these regions, and decreasing during the dark cycle, when WFA+ PNN densities are high. Similar diurnal rhythms were also observed in the infralimbic cortex superficial layers $(\boldsymbol{D})$, prelimbic cortex superficial layers $(\boldsymbol{E})$, infralimbic cortex deep layers $(\boldsymbol{F})$, and prelimbic cortex deep layers $(\boldsymbol{G})$. Error bars represent SD.

examining this process. However, a significant limitation is that our current data show associations but do not demonstrate mechanistic effects of cathepsin-S expression rhythms on PNN rhythms. Our data showing diurnal rhythms of cathepsin-S expression represents the first step in testing a broad range of proteases from several cell types. Circadian regulation of PNNs is likely to consist of a complex molecular signaling system involving multiple proteases and ECM molecules, encompassing several cell types. Future studies focused on circadian expression of specific PNN components, matrix proteases and sulfotransferases will provide insight into the mechanisms underlying circadian PNN modification and direct effects on memory processing.

\section{TOD in human postmortem subjects as a proxy for diurnal} rhythms (ZT)

Human postmortem studies have successfully used TOD as a proxy for diurnal rhythms (approximate ZT), to study diurnal rhythms of gene and protein expression in human brain. An obvious limitation is that TOD represents a single measure per subject at a specific time point, rather than repeated measures across time. However, several human studies demonstrated predicted rhythmic 
A

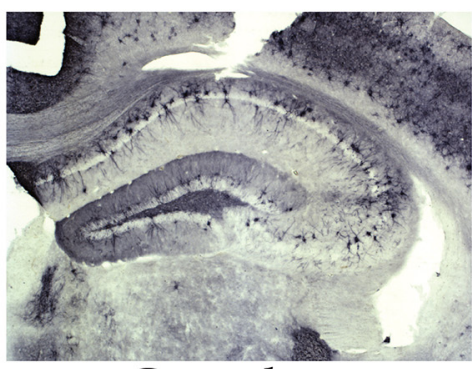

Control

D

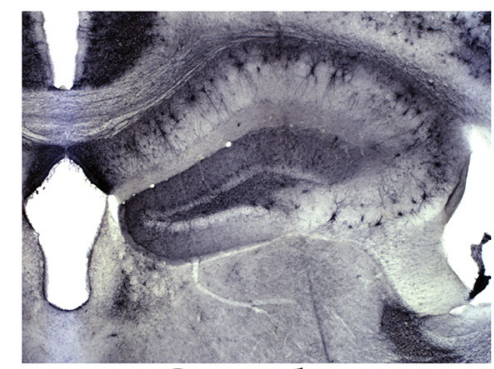

Control
B

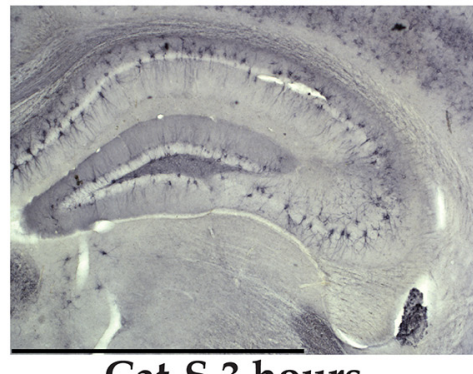

Cat-S 3 hours

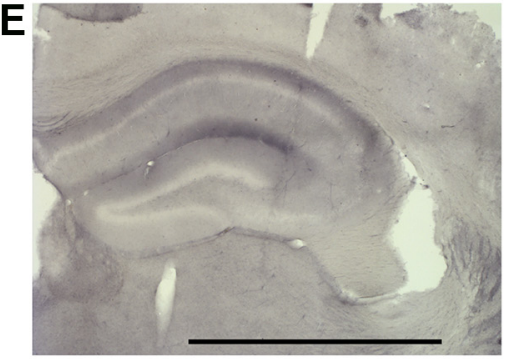

Cat-S 24 hours
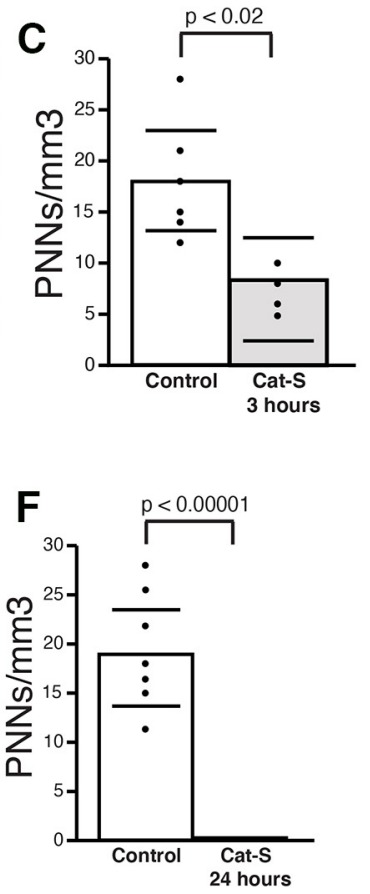
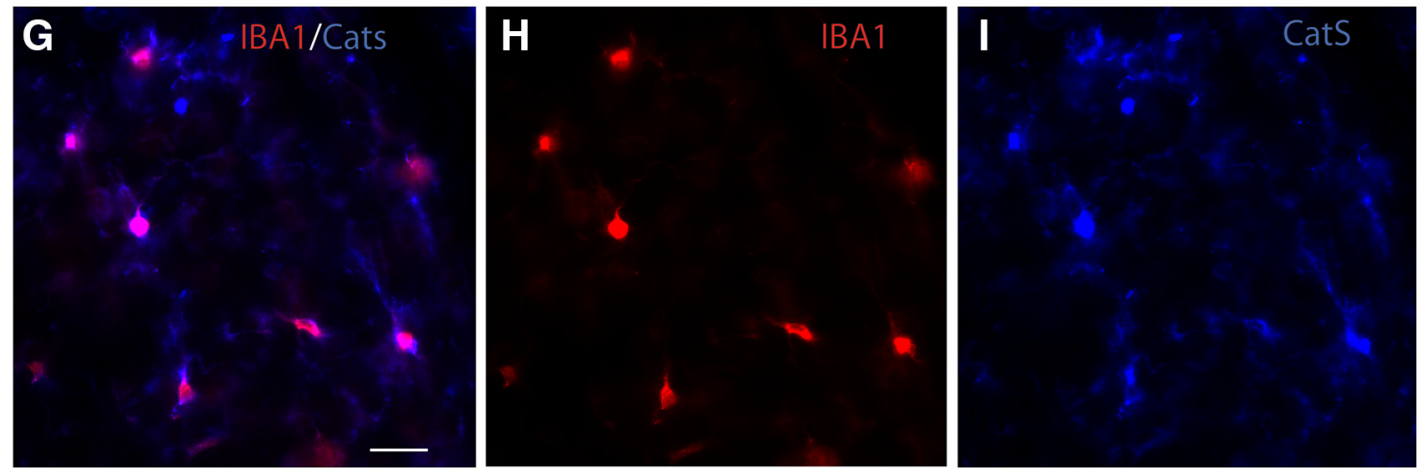

CatS-IBA1 cells = CatS only cells

J Hippocampus

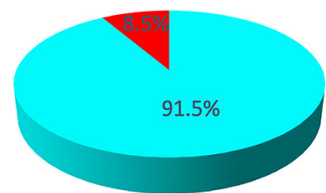

K Amygdala

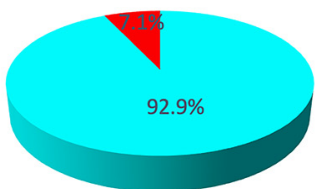

L IL \& PL Cortex

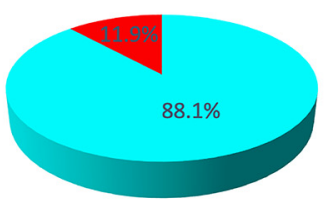

M Habenula

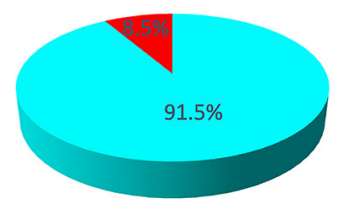

Thalamic

$\mathbf{N}$ Reticular Nucleus

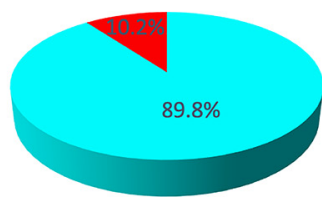

Figure 10. Cathepsin-S is expressed in microglia and eliminates PNN labeling. Significant reduction in WFA+ PNNs is observed after $3 \mathrm{~h}$ of cathepsin-S incubation $(\boldsymbol{A}-\boldsymbol{C})$ and a complete absence of PNN labeling after $24 \mathrm{~h}(\boldsymbol{D}-\boldsymbol{F})$. Error bars represent $95 \%$ confidence interval. Scale bars $=1000 \mu \mathrm{m}$. Dual fluorescence immunohistochemistry demonstrated that the vast majority of cathepsin-S-IR cells in the mouse hippocampus co-express the microglial marker IBA1 (G-N). Scale bar $=50 \mu \mathrm{m}$.

expression of clock genes in several brain regions, and of SST in the amygdala (Li et al., 2013; Bunney et al., 2015; Chen et al., 2016; Pantazopoulos et al., 2017). Importantly, rhythmic patterns, such as peak phase relationships between clock molecules, demonstrated in human were consistent with those reported in rodents, including staggered phase relationship between Per1, Per2, and
Per3 genes (Lamont et al., 2005; Ramanathan et al., 2010; Albrecht et al., 2013; Li et al., 2013). Molecular rhythms reported in the human cortex have been independently replicated, providing further support for the validity of this approach (Li et al., 2013; Chen et al., 2016). The WFA+ PNN rhythms observed in the amygdala nocturnal mice (Figs. 1, 6) are antiphase to the PNN rhythms observed in 

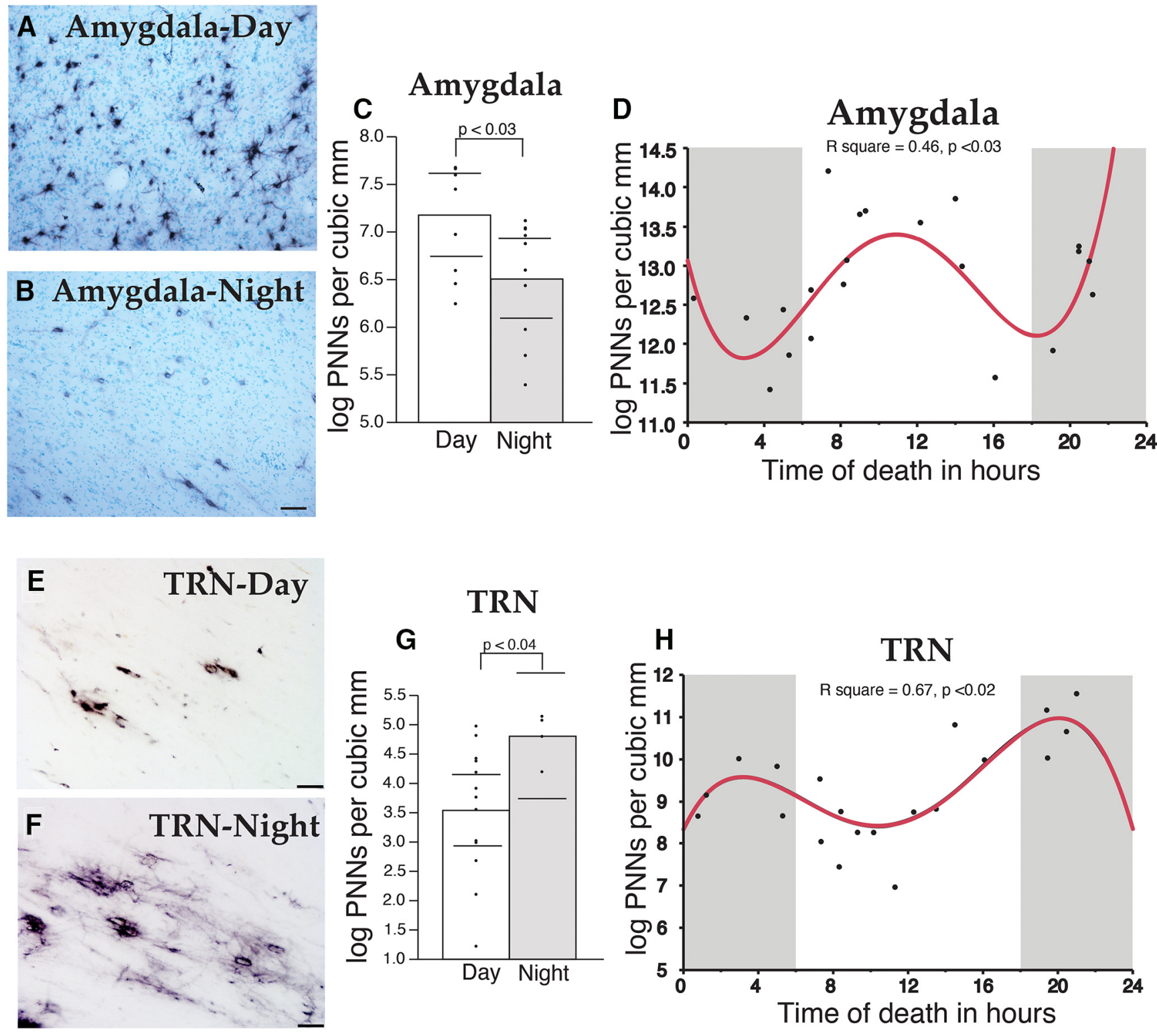

Figure 11. Diurnal rhythms of PNNs in the human brain. WFA+ PNN numbers vary with TOD in the human brain. Photomicrograph depicting PNN labeling by WFA lectin in the human amygdala during the day $(\boldsymbol{A})$ and at night (B). WFA+ PNNs displayed a significant day/night difference in the human amygdala $(\boldsymbol{C})$, with peaks PNN numbers at noon and midnight, and troughs at 4 A.M. and 8 P.M. D, Photomicrograph depicting PNN labeling in the human TRN during the day $(\boldsymbol{E})$ and at night $(\boldsymbol{F})$. Significant day/night differences were observed in total numbers of WFA+ PNNs in the TRN $(\boldsymbol{G})$. Quartic regression plots revealed a dual peak rhythm in the TRN that is antiphase to the rhythm observed in the amygdala $(\boldsymbol{H})$. Error bars represent $95 \%$ confidence intervals.

diurnal human subjects in the same region (Fig. 11), providing further support for the approach of using TOD to analyze rhythmic relationships in human postmortem samples.

\section{Implications for synaptic plasticity and memory consolidation}

Several hypotheses have been put forth to link wake/ sleep cycles to synaptic mechanisms underlying memory consolidation. For instance, studies from Tononi and Cirelli support the synaptic homeostasis hypothesis of sleep (Tononi and Cirelli, 2006, 2014). Briefly, neurons form and strengthen many new synapses during wakefulness, as organisms interact with their environment and encode new memories. During sleep, when the active encoding process is offline, synapses are downscaled, to enhance the signal-to-noise ratio, thus improving memory function (Tononi and Cirelli, 2006, 2014). Consistent with this hypothesis, decreases of dendritic spines and synapses during sleep have been reported in sensory and motor cortical regions (Maret et al., 2011; de Vivo et al., 2017). An alternative theory, suggested by Rasch and Born, postulates that memories are reorganized during slow wave sleep in a process called systemic consolidation (Rasch and Born, 2013). During systemic consolidation, memory representations are reactivated and 
transferred from short-term storage sites, such as the hippocampus, into long-term storage in neocortical areas where they are integrated into existing schemas (Rasch and Born, 2013). Memories are then strengthened in these long-term storage areas during REM sleep, in a process called synaptic consolidation, while the short-term storage memories are removed via synaptic pruning (Rasch and Born, 2013).

We speculate that diurnal molecular modifications of PNNs may contribute to memory formation and consolidation mechanisms during the wake/sleep cycle, favoring activity-driven synaptogenesis and synaptic refinement, respectively. For instance, our results on the effects of 5-h sleep deprivation on WFA+ PNNs in the mouse hippocampus are consistent with reports that $5 \mathrm{~h}$ of sleep deprivation prevents changes in dendritic spine densities in the hippocampus occurring during sleep (Havekes et al., 2016; Raven et al., 2019; Spano et al., 2019; Gisabella et al., 2020). PNN rhythms observed in our study may reflect ongoing systemic and synaptic consolidation during sleep proposed by Rasch and Born (Rasch and Born, 2013). For instance, WFA+ PNNs changes in mice the hippocampus are more active during wakefulness, as suggested by enhanced LTP in this region during the night (Chaudhury et al., 2005). Regional differences in PNN rhythms may also reflect phase differences in molecular clock rhythms of these regions. Region specific rhythms in the clock protein Per2 have been described previously in rodents and humans (Lamont et al., 2005; Li et al., 2013; Harbour et al., 2014; Chen et al., 2016).

Recent evidence shows that cathepsin-S deletion in knock-out mice contributes to failure to downscale synapses during sleep (Hayashi et al., 2013b). In these mice, reduced EEG $\delta$ wave power and failure to reduce amplitude and frequency of action potentials and to reduce dendritic spines during sleep supports a role for cathepsin-S in downscaling synaptic strength during sleep (Hayashi et al., 2013b). Our results show that rhythms of cathepsin-S expression are antiphase with respect to WFA+ PNNs rhythms, i.e., high cathepsin-S expression is associated with low WFA+ PNN numbers, and that cathepsin-S reduces WFA+ PNN labeling. Together, these findings suggest that increased cathepsin-S during sleep may represent one of several molecules that contribute to modifying PNN composition. In turn, such modifications may contribute to synaptic downscaling and remodeling during memory consolidation (Fig. 12). This hypothesis is supported by evidence for the involvement of the microglial circadian molecular clock in the regulation of microglial morphology, immune response, and synaptic regulation (Hayashi et al., 2013a,b; Fonken et al., 2015). Our results suggest an additional circadian role for microglia in synaptic regulation, through PNN modification potentially modulating memory consolidation processes.

Circadian rhythms in PNN composition may also be regulated by proteases and CSPG production from several cell types including astrocytes and neurons, which produce many of the core PNN components as well as endogenous proteases known to modify PNNs (Pantazopoulos and

\section{WFA+ PNN decreases coincide with reported daytime decreases of LTP}

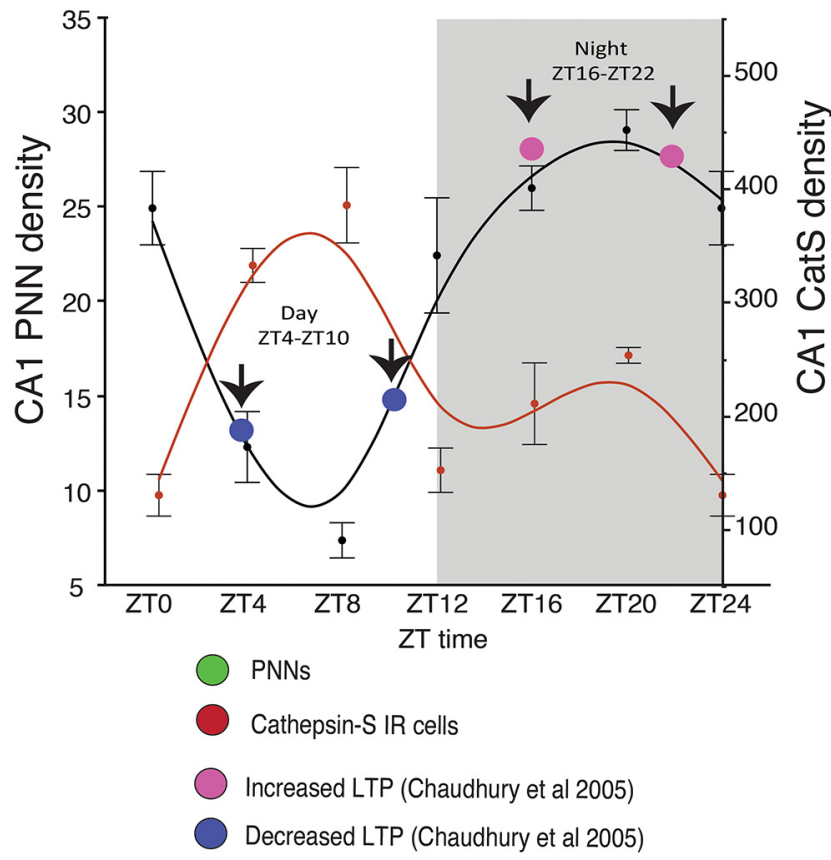

Figure 12. Microglial expression of cathepsin-S may modify PNNs to allow for memory consolidation during sleep. In the mouse hippocampal sector CA1, diurnal rhythms in the numerical density of WFA + PNNs decreases during the day as mice sleep, reaching the lowest density in WFA+ PNN numbers between ZT4-ZT10 (green curved line). This coincides with the peak expression of cathepsin-S (red curved line) and the reported daytime decrease in LTP (blue circles; from Chaudhury et al., 2005). In comparison, the numerical density of WFA+ PNNs peaks during the dark at $\sim$ ZT20 during the active period for nocturnal mice, coinciding with the low point of cathepsin-S immunoreactivity in this region as well as the reported increase in LTP at night in mice (pink circles; from Chaudhury et al., 2005). These results suggest that cathepsin-S modifies PNN composition, coinciding with decreased TLP during sleep, to allow for memory consolidation, and PNN composition is restored during the active wake periods to allow for optimal encoding of novel information.

Berretta, 2016; Miyata and Kitagawa, 2017; Bozzelli et al., 2018). Furthermore, although our evidence suggests that circadian rhythms in PNN composition may contribute to synaptic regulation during sleep, we do not demonstrate a mechanistic effect on synaptic regulation or memory consolidation. PNN circadian rhythms may be involved in other processes such as resolution of oxidative stress during sleep. Several studies suggest that sleep deprivation contributes to increased oxidative stress in in the brain (Silva et al., 2004; Ramanathan and Siegel, 2011; Alzoubi et al., 2012; Harkness et al., 2019), and PNNs are critically involved in protecting fast-firing neurons from oxidative stress (Cabungcal et al., 2013). Thus, rhythms in PNN composition may reflect periods of reduced neuronal activity and resolution of oxidative stress during sleep. A recent study reporting increased oxidative stress in parvalbumin (PVB) neurons together with increased WFA labeling of PNNs following 
sleep deprivation supports this hypothesis (Harkness et al., 2019).

\section{Implications for psychiatric disorders}

In the present study, we focused on brain regions involved in emotional memory processing and implicated in psychiatric disorders (Vyas et al., 2002; Sartorius et al., 2010; Li et al., 2011; Mahan and Ressler, 2012; Mauney et al., 2013; Meyer et al., 2014; Pantazopoulos et al., 2017; Wells et al., 2017). Diurnal rhythms of PNNs in human subjects have broad implications for psychiatric disorders. PNN deficits have been reported by several groups in the amygdala, entorhinal cortex, hippocampus, prefrontal cortex, and TRN in schizophrenia and bipolar disorder (Pantazopoulos et al., 2010, 2014, 2015; Mauney et al., 2013; Enwright et al., 2016; Steullet et al., 2018). Disruption of PNNs in these disorders may alter rhythms of synaptic plasticity and in turn contribute to shared synaptic deficits (Penzes et al., 2011; Glausier and Lewis, 2013; Shelton et al., 2015; MacDonald et al., 2017). Such deficits may arise from disrupted memory consolidation processes allowing for decreased synaptic formation and/or increased synaptic pruning in brain regions involved in emotional memory processing.

Abnormalities in sleep and circadian rhythms have also been consistently reported in these disorders (McClung, 2013; Manoach et al., 2016; Pantazopoulos et al., 2017; Seney et al., 2019). Decreased sleep spindles, generated by the TRN, and memory consolidation deficits are emerging as consistent characteristics of schizophrenia (Ferrarelli et al., 2007; Manoach et al., 2010, 2014). Decreased sleep spindles have been reported in several independent studies, including in unmedicated patients with schizophrenia, and in first-degree relatives, suggesting that this represents a core genetic component of the disease rather than medication effects or consequence of disease progression (Ferrarelli et al., 2007; Manoach et al., 2010, 2014). Disruption of WFA+ PNN rhythms in subjects with schizophrenia may contribute to sleep spindle and memory consolidation deficits in several ways. WFA + PNNs regulate firing rates of neurons expressing PVB, including those in the TRN that generate sleep spindles (Csillik et al., 2005; Katsuki et al., 2017). Furthermore, decreases of PVB neurons were detected in the TRN of subjects with schizophrenia (Steullet et al., 2018). PNNs protect PVB neurons from oxidative stress (Cabungcal et al., 2013), thus disruption of PNN rhythms may leave PVB neurons more susceptible to accumulation of oxidative damage during sleep, resulting in loss of PVB neurons in subjects with schizophrenia (Steullet et al., 2018). PVB deficits in TRN function have been proposed by several groups to contribute to memory consolidation deficits in schizophrenia (Manoach et al., 2016; Ferrarelli and Tononi, 2017). Disrupted PNN rhythms in the TRN may contribute to a decreased ability of this region to generate sleep spindles and, in turn, memory consolidation deficits. In addition, disrupted PNN rhythm composition by cathepsin-S in expression from microglia in subjects with schizophrenia may contribute to memory consolidation deficits through disruption of local synaptic downscaling and reorganization proposed to occur during sleep (Tononi and Cirelli, 2006, 2014; Rasch and Born, 2013). Cathepsin-S knock-out mice, in which diurnal rhythms of dendritic spine density were reported (Hayashi et al., 2013b), also display deficits in social interaction and novel object recognition (Takayama et al., 2017), supporting the hypothesis that cathepsin-S rhythms regulate key roles of PNNs in memory processing and social behaviors that are disrupted in subjects with schizophrenia.

Our findings may also be relevant to the pathophysiology of PTSD. PNNs are strongly involved in fear memory processing, which is enhanced in this disorder (for review, see Parsons and Ressler, 2013; see also Gogolla et al., 2009; Banerjee et al., 2017). Sleep deprivation has been proposed as an early therapeutic approach for PTSD following a traumatic experience (Kuriyama et al., 2010; Cohen et al., 2012, 2017). Disruption of molecular processes involved in PNN rhythms may represent one of the potential mechanisms through which sleep deprivation may impact memory consolidation as a possible therapeutic approach for alleviating the strength of fear memories contributing to PTSD.

In summary, we provide evidence for diurnal and circadian rhythms of WFA+ PNN numbers in the human and rodent brain, suggesting that their composition is modified on a daily basis. Rhythms in PNN composition may be mediated in part by cathepsin-S expression originating from microglia. These rhythms may contribute to decreased LTP reported during sleep in the hippocampus, suggesting a key process through which multiple cell types including microglia modify PNNs to allow for to memory consolidation.

\section{References}

Albrecht A, Thiere M, Bergado-Acosta JR, Poranzke J, Müller B, Stork O (2013) Circadian modulation of anxiety: a role for somatostatin in the amygdala. PLoS One 8:e84668.

Alzoubi KH, Khabour OF, Rashid BA, Damaj IM, Salah HA (2012) The neuroprotective effect of vitamin $\mathrm{E}$ on chronic sleep deprivation-induced memory impairment: the role of oxidative stress. Behav Brain Res 226:205-210.

Amaral DG, Price JL, Pitkanen A, Carmichael ST (1992) Anatomical organization of the primate amygdaloid complex. In: The amygdala: neurobiological aspects of emotion, memory, and mental dysfunction (Aggleton JP, ed). New York: Wiley.

Arnold MA, Reppert SM, Rorstad OP, Sagar SM, Keutmann HT, Perlow MJ, Martin JB (1982) Temporal patterns of somatostatin immunoreactivity in the cerebrospinal fluid of the rhesus monkey: effect of environmental lighting. J Neurosci 2:674-680.

Baig S, Wilcock GK, Love S (2005) Loss of perineuronal net N-acetylgalactosamine in Alzheimer's disease. Acta Neuropathol 110:393401.

Bajor M, Kaczmarek L (2013) Proteolytic remodeling of the synaptic cell adhesion molecules (CAMs) by metzincins in synaptic plasticity. Neurochem Res 38:1113-1121.

Balmer TS, Carels VM, Frisch JL, Nick TA (2009) Modulation of perineuronal nets and parvalbumin with developmental song learning. J Neurosci 29:12878-12885.

Banerjee SB, Gutzeit VA, Baman J, Aoued HS, Doshi NK, Liu RC, Ressler KJ (2017) Perineuronal nets in the adult sensory cortex are necessary for fear learning. Neuron 95:169-179.e3.

Berelowitz M, Perlow MJ, Hoffman HJ, Frohman LA (1981) The diurnal variation of immunoreactive thyrotropin-releasing hormone 
and somatostatin in the cerebrospinal fluid of the rhesus monkey. Endocrinology 109:2102-2109.

Berretta S, Pantazopoulos H, Lange N (2007) Neuron numbers and volume of the amygdala in subjects diagnosed with bipolar disorder or schizophrenia. Biol Psychiatry 62:884-893.

Blacktop JM, Sorg BA (2019) Perineuronal nets in the lateral hypothalamus area regulate cue-induced reinstatement of cocaineseeking behavior. Neuropsychopharmacology 44:850-858.

Bolós M, Perea JR, Terreros-Roncal J, Pallas-Bazarra N, JuradoArjona J, Ávila J, Llorens-Martín M (2018) Absence of microglial CX3CR1 impairs the synaptic integration of adult-born hippocampal granule neurons. Brain Behav Immun 68:76-89.

Bozzelli PL, Alaiyed S, Kim E, Villapol S, Conant K (2018) Proteolytic remodeling of perineuronal nets: effects on synaptic plasticity and neuronal population dynamics. Neural Plast 2018:5735789.

Brakebusch C, Seidenbecher Cl, Asztely F, Rauch U, Matthies H, Meyer H, Krug M, Böckers TM, Zhou X, Kreutz MR, Montag D, Gundelfinger ED, Fässler R (2002) Brevican-deficient mice display impaired hippocampal CA1 long-term potentiation but show no obvious deficits in learning and memory. Mol Cell Biol 22:74177427

Brown TE, Wilson AR, Cocking DL, Sorg BA (2009) Inhibition of matrix metalloproteinase activity disrupts reconsolidation but not consolidation of a fear memory. Neurobiol Learn Mem 91:66-72.

Bukalo O, Schachner M, Dityatev A (2001) Modification of extracellular matrix by enzymatic removal of chondroitin sulfate and by lack of tenascin-R differentially affects several forms of synaptic plasticity in the hippocampus. Neuroscience 104:359-369.

Bunney BG, Li JZ, Walsh DM, Stein R, Vawter MP, Cartagena P, Barchas JD, Schatzberg AF, Myers RM, Watson SJ, Akil H, Bunney WE (2015) Circadian dysregulation of clock genes: clues to rapid treatments in major depressive disorder. Mol Psychiatry 20:48-55.

Cabungcal JH, Steullet $P$, Morishita $H$, Kraftsik R, Cuenod M, Hensch TK, Do KQ (2013) Perineuronal nets protect fast-spiking interneurons against oxidative stress. Proc Natl Acad Sci USA 110:9130-9135.

Caterson B, Griffin J, Mahmoodian F, Sorrell JM (1990) Monoclonal antibodies against chondroitin sulphate isomers: their use as probes for investigating proteoglycan metabolism. Biochem Soc Trans 18:820-823.

Chaudhury D, Wang LM, Colwell CS (2005) Circadian regulation of hippocampal long-term potentiation. J Biol Rhythms 20:225-236.

Chen CY, Logan RW, Ma T, Lewis DA, Tseng GC, Sibille E, McClung CA (2016) Effects of aging on circadian patterns of gene expression in the human prefrontal cortex. Proc Natl Acad Sci USA 113:206-211.

Coggeshall RE, Lekan HA (1996) Methods for determining number of cells and synapses: a case for more uniform standard of review. J Comp Neurol 364:6-15.

Cohen S, Kozlovsky N, Matar MA, Kaplan Z, Zohar J, Cohen H (2012) Post-exposure sleep deprivation facilitates correctly timed interactions between glucocorticoid and adrenergic systems, which attenuate traumatic stress responses. Neuropsychopharmacology 37:2388-2404.

Cohen S, Kaplan Z, Zohar J, Cohen H (2017) Preventing sleep on the first resting phase following a traumatic event attenuates anxietyrelated responses. Behav Brain Res 320:450-456.

Crapser JD, Ochaba J, Soni N, Reidling JC, Thompson LM, Green KN (2020) Microglial depletion prevents extracellular matrix changes and striatal volume reduction in a model of Huntington's disease. Brain 143:266-288.

Csillik B, Mihály A, Krisztin-Péva B, Chadaide Z, Samsam M, Knyihár-Csillik E, Fenyo R (2005) GABAergic parvalbumin-immunoreactive large calyciform presynaptic complexes in the reticular nucleus of the rat thalamus. J Chem Neuroanat 30:17-26.

de Vivo L, Bellesi M, Marshall W, Bushong EA, Ellisman MH, Tononi G, Cirelli C (2017) Ultrastructural evidence for synaptic scaling across the wake/sleep cycle. Science 355:507-510.
Dingess PM, Harkness JH, Slaker M, Zhang Z, Wulff SS, Sorg BA, Brown TE (2018) Consumption of a high-fat diet alters perineuronal nets in the prefrontal cortex. Neural Plast 2018:2108373.

Dorph-Petersen KA, Lewis DA (2011) Stereological approaches to identifying neuropathology in psychosis. Biol Psychiatry 69:113126.

Dumont M, Macchi MM, Carrier J, Lafrance C, Hébert M (1999) Time course of narrow frequency bands in the waking EEG during sleep deprivation. Neuroreport 10:403-407.

Enwright JF, Sanapala S, Foglio A, Berry R, Fish KN, Lewis DA (2016) Reduced labeling of parvalbumin neurons and perineuronal nets in the dorsolateral prefrontal cortex of subjects with schizophrenia. Neuropsychopharmacology 41:2206-2214.

Ferrarelli F, Tononi G (2017) Reduced sleep spindle activity point to a TRN-MD thalamus-PFC circuit dysfunction in schizophrenia. Schizophr Res 180:36-43.

Ferrarelli F, Huber R, Peterson MJ, Massimini M, Murphy M, Riedner BA, Watson A, Bria P, Tononi G (2007) Reduced sleep spindle activity in schizophrenia patients. Am J Psychiatry 164:483-492.

Fonken LK, Frank MG, Kitt MM, Barrientos RM, Watkins LR, Maier SF (2015) Microglia inflammatory responses are controlled by an intrinsic circadian clock. Brain Behav Immun 45:171-179.

Foscarin S, Raha-Chowdhury R, Fawcett JW, Kwok JCF (2017) Brain ageing changes proteoglycan sulfation, rendering perineuronal nets more inhibitory. Aging (Albany NY) 9:1607-1622.

Galtrey CM, Fawcett JW (2007) The role of chondroitin sulfate proteoglycans in regeneration and plasticity in the central nervous system. Brain Res Rev 54:1-18.

Ganguly K, Rejmak E, Mikosz M, Nikolaev E, Knapska E, Kaczmarek L (2013) Matrix metalloproteinase (MMP) 9 transcription in mouse brain induced by fear learning. J Biol Chem 288:20978-20991.

Geissler M, Gottschling C, Aguado A, Rauch U, Wetzel CH, Hatt H, Faissner A (2013) Primary hippocampal neurons, which lack four crucial extracellular matrix molecules, display abnormalities of synaptic structure and function and severe deficits in perineuronal net formation. J Neurosci 33:7742-7755.

Gisabella B, Farah S, Peng X, Burgos-Robles A, Lim SH, Goosens KA (2016) Growth hormone biases amygdala network activation after fear learning. Transl Psychiatry 6:e960.

Gisabella B, Scammell T, Bandaru SS, Saper CB (2020) Regulation of hippocampal dendritic spines following sleep deprivation. J Comp Neurol 528:380-388.

Glausier JR, Lewis DA (2013) Dendritic spine pathology in schizophrenia. Neuroscience 251:90-107.

Gogolla N, Caroni P, Lüthi A, Herry C (2009) Perineuronal nets protect fear memories from erasure. Science 325:1258-1261.

Gundersen HJ, Jensen EB, Kiêu K, Nielsen J (1999) The efficiency of systematic sampling in stereology-reconsidered. J Microsc 193:199-211.

Harbour VL, Weigl Y, Robinson B, Amir S (2014) Phase differences in expression of circadian clock genes in the central nucleus of the amygdala, dentate gyrus, and suprachiasmatic nucleus in the rat. PLoS One 9:e103309.

Harkness JH, Bushana PN, Todd RP, Clegern WC, Sorg BA, Wisor JP (2019) Sleep disruption elevates oxidative stress in parvalbumin-positive cells of the rat cerebral cortex. Sleep 42:zsy201.

Havekes R, Park AJ, Tolentino RE, Bruinenberg VM, Tudor JC, Lee Y, Hansen RT, Guercio LA, Linton E, Neves-Zaph SR, Meerlo P, Baillie GS, Houslay MD, Abel T (2016) Compartmentalized PDE4A5 signaling impairs hippocampal synaptic plasticity and long-term memory. J Neurosci 36:8936-8946.

Hayashi Y, Koyanagi S, Kusunose N, Takayama F, Okada R, Wu Z, Ohdo S, Nakanishi H (2013a) Diurnal spatial rearrangement of microglial processes through the rhythmic expression of P2Y12 receptors. Neurol Disord 1:1-7.

Hayashi Y, Koyanagi S, Kusunose N, Okada R, Wu Z, Tozaki-Saitoh H, Ukai K, Kohsaka S, Inoue K, Ohdo S, Nakanishi H (2013b) The intrinsic microglial molecular clock controls synaptic strength via the circadian expression of cathepsin S. Sci Rep 3:2744. 
Hofman MA (2003) Circadian oscillations of neuropeptide expression in the human biological clock. J Comp Physiol A Neuroethol Sens Neural Behav Physiol 189:823-831.

Hou Y, Huang Q, Prakash R, Chaudhury S (2013) Infrequent near death experiences in severe brain injury survivors - A quantitative and qualitative study. Ann Indian Acad Neurol 16:75-81.

I wata O, Okamura H, Saitsu H, Saikusa M, Kanda H, Eshima N, Iwata S, Maeno Y, Matsuishi T (2013) Diurnal cortisol changes in newborn infants suggesting entrainment of peripheral circadian clock in utero and at birth. J Clin Endocrinol Metab 98:E25-E32.

Katsuki F, McNally JM, Thankachan S, McKenna JT, Brown RE, Strecker RE, McCarley RW (2017) Optogenetic manipulation of parvalbumin containing GABAergic neurons in the thalamic reticular nucleus alters declarative and non-declarative memories in mice. Sleep 40:A80-A81.

Kuriyama K, Soshi T, Kim Y (2010) Sleep deprivation facilitates extinction of implicit fear generalization and physiological response to fear. Biol Psychiatry 68:991-998.

Kurokawa T, Tsuda M, Sugino Y (1976) Purification and characterization of a lectin from Wisteria floribunda seeds. J Biol Chem 251:5686-5693.

Lamont EW, Robinson B, Stewart J, Amir S (2005) The central and basolateral nuclei of the amygdala exhibit opposite diurnal rhythms of expression of the clock protein Period2. Proc Natl Acad Sci USA 102:4180-4184.

Lasek AW, Chen H, Chen WY (2018) Releasing addiction memories trapped in perineuronal nets. Trends Genet 34:197-208.

Li B, Piriz J, Mirrione M, Chung C, Proulx CD, Schulz D, Henn F, Malinow R (2011) Synaptic potentiation onto habenula neurons in the learned helplessness model of depression. Nature 470:535539.

Li JZ, Bunney BG, Meng F, Hagenauer MH, Walsh DM, Vawter MP, Evans SJ, Choudary PV, Cartagena P, Barchas JD, Schatzberg AF, Jones EG, Myers RM, Watson SJ Jr, Akil H, Bunney WE (2013) Circadian patterns of gene expression in the human brain and disruption in major depressive disorder. Proc Natl Acad Sci USA 110:9950-9955.

Lim AS, Kowgier M, Yu L, Buchman AS, Bennett DA (2013) Sleep fragmentation and the risk of incident Alzheimer's disease and cognitive decline in older persons. Sleep 36:1027-1032.

MacDonald ML, Alhassan J, Newman JT, Richard M, Gu H, Kelly RM, Sampson AR, Fish KN, Penzes P, Wills ZP, Lewis DA, Sweet RA (2017) Selective loss of smaller spines in schizophrenia. Am J Psychiatry 174:586-594.

Maeda N (2010) Structural variation of chondroitin sulfate and its roles in the central nervous system. Cent Nerv Syst Agents Med Chem 10:22-31.

Mahan AL, Ressler KJ (2012) Fear conditioning, synaptic plasticity and the amygdala: implications for posttraumatic stress disorder. Trends Neurosci 35:24-35.

Manoach DS, Thakkar KN, Stroynowski E, Ely A, McKinley SK, Wamsley E, Djonlagic I, Vangel MG, Goff DC, Stickgold R (2010) Reduced overnight consolidation of procedural learning in chronic medicated schizophrenia is related to specific sleep stages. $J$ Psychiatr Res 44:112-120.

Manoach DS, Demanuele C, Wamsley EJ, Vangel M, Montrose DM, Miewald J, Kupfer D, Buysse D, Stickgold R, Keshavan MS (2014) Sleep spindle deficits in antipsychotic-naïve early course schizophrenia and in non-psychotic first-degree relatives. Front Hum Neurosci 8:762.

Manoach DS, Pan JQ, Purcell SM, Stickgold R (2016) Reduced sleep spindles in schizophrenia: a treatable endophenotype that links risk genes to impaired cognition? Biol Psychiatry 80:599-608.

Maret S, Faraguna U, Nelson AB, Cirelli C, Tononi G (2011) Sleep and waking modulate spine turnover in the adolescent mouse cortex. Nat Neurosci 14:1418-1420.

Mauney SA, Athanas KM, Pantazopoulos H, Shaskan N, Passeri E, Berretta S, Woo TU (2013) Developmental pattern of perineuronal nets in the human prefrontal cortex and their deficit in schizophrenia. Biol Psychiatry 74:427-435.
McClung CA (2013) How might circadian rhythms control mood? Let me count the ways. Biol Psychiatry 74:242-249.

Meyer RM, Burgos-Robles A, Liu E, Correia SS, Goosens KA (2014) A ghrelin-growth hormone axis drives stress-induced vulnerability to enhanced fear. Mol Psychiatry 19:1284-1294.

Miyata S, Kitagawa $\mathrm{H}$ (2017) Formation and remodeling of the brain extracellular matrix in neural plasticity: roles of chondroitin sulfate and hyaluronan. Biochim Biophys Acta Gen Subj 1861:24202434.

Miyata S, Komatsu Y, Yoshimura Y, Taya C, Kitagawa H (2012) Persistent cortical plasticity by upregulation of chondroitin 6-sulfation. Nat Neurosci 15:414-422, S1-2.

Monk TH, Buysse DJ, Reynolds CF 3rd, Berga SL, Jarrett DB, Begley AE, Kupfer DJ (1997) Circadian rhythms in human performance and mood under constant conditions. J Sleep Res 6:9-18.

Morawski M, Brückner G, Jäger C, Seeger G, Arendt T (2010) Neurons associated with aggrecan-based perineuronal nets are protected against tau pathology in subcortical regions in Alzheimer's disease. Neuroscience 169:1347-1363.

Muir EM, Adcock KH, Morgenstern DA, Clayton R, von Stillfried N, Rhodes K, Ellis C, Fawcett JW, Rogers JH (2002) Matrix metalloproteases and their inhibitors are produced by overlapping populations of activated astrocytes. Brain Res Mol Brain Res 100:103117.

Nagy V, Bozdagi O, Matynia A, Balcerzyk M, Okulski P, Dzwonek J, Costa RM, Silva AJ, Kaczmarek L, Huntley GW (2006) Matrix metalloproteinase-9 is required for hippocampal late-phase long-term potentiation and memory. J Neurosci 26:1923-1934.

Nagy V, Bozdagi O, Huntley GW (2007) The extracellular protease matrix metalloproteinase- 9 is activated by inhibitory avoidance learning and required for long-term memory. Learn Mem 14:655664.

Pantazopoulos H, Berretta S (2016) In sickness and in health: perineuronal nets and synaptic plasticity in psychiatric disorders. Neural Plast 2016:9847696.

Pantazopoulos H, Lange N, Baldessarini RJ, Berretta S (2007) Parvalbumin neurons in the entorhinal cortex of subjects diagnosed with bipolar disorder or schizophrenia. Biol Psychiatry 61:640-652.

Pantazopoulos H, Woo TU, Lim MP, Lange N, Berretta S (2010) Extracellular matrix-glial abnormalities in the amygdala and entorhinal cortex of subjects diagnosed with schizophrenia. Arch Gen Psychiatry 67:155-166.

Pantazopoulos H, Sawyer C, Heckers S, Berretta S, Markota M (2014) Chondroitin sulfate proteoglycan abnormalities in the hippocampus of subjects with schizophrenia. Neuropsychopharmacology 39: S298-S299.

Pantazopoulos H, Markota M, Jaquet F, Ghosh D, Wallin A, Santos A, Caterson B, Berretta S (2015) Aggrecan and chondroitin-6-sulfate abnormalities in schizophrenia and bipolar disorder: a postmortem study on the amygdala. Transl Psychiatry 5:e496.

Pantazopoulos H, Wiseman JT, Markota M, Ehrenfeld L, Berretta S (2017) Decreased numbers of somatostatin-expressing neurons in the amygdala of subjects with bipolar disorder or schizophrenia: relationship to circadian rhythms. Biol Psychiatry 81:536-547.

Parsons RG, Ressler KJ (2013) Implications of memory modulation for post-traumatic stress and fear disorders. Nat Neurosci 16:146153.

Penzes P, Cahill ME, Jones KA, VanLeeuwen JE, Woolfrey KM (2011) Dendritic spine pathology in neuropsychiatric disorders. Nat Neurosci 14:285-293.

Petanceska S, Canoll P, Devi LA (1996) Expression of rat cathepsin S in phagocytic cells. J Biol Chem 271:4403-4409.

Pizzorusso T, Medini P, Berardi N, Chierzi S, Fawcett JW, Maffei L (2002) Reactivation of ocular dominance plasticity in the adult visual cortex. Science 298:1248-1251.

Porter S, Clark IM, Kevorkian L, Edwards DR (2005) The ADAMTS metalloproteinases. Biochem J 386:15-27. 
Ramanathan L, Siegel JM (2011) Sleep deprivation under sustained hypoxia protects against oxidative stress. Free Radic Biol Med 51:1842-1848.

Ramanathan C, Stowie A, Smale L, Nunez A (2010) PER2 rhythms in the amygdala and bed nucleus of the stria terminalis of the diurnal grass rat (Arvicanthis niloticus). Neurosci Lett 473:220-223.

Rasch B, Born J (2013) About sleep's role in memory. Physiol Rev 93:681-766.

Raven F, Meerlo P, Van der Zee EA, Abel T, Havekes R (2019) A brief period of sleep deprivation causes spine loss in the dentate gyrus of mice. Neurobiol Learn Mem 160:83-90.

Rubinow DR (1986) Cerebrospinal fluid somatostatin and psychiatric illness. Biol Psychiatry 21:341-365.

Sartorius A, Kiening KL, Kirsch $\mathrm{P}$, von Gall CC, Haberkorn U, Unterberg AW, Henn FA, Meyer-Lindenberg A (2010) Remission of major depression under deep brain stimulation of the lateral habenula in a therapy-refractory patient. Biol Psychiatry 67:e9-e11.

Schmal C, Reimann P, Staiger D (2013) A circadian clock-regulated toggle switch explains AtGRP7 and AtGRP8 oscillations in Arabidopsis thaliana. PLoS Comput Biol 9:e1002986.

Schnell SA, Staines WA, Wessendorf MW (1999) Reduction of lipofuscin-like autofluorescence in fluorescently labeled tissue. J Histochem Cytochem 47:719-730.

Segall LA, Milet A, Tronche F, Amir S (2009) Brain glucocorticoid receptors are necessary for the rhythmic expression of the clock protein, PERIOD2, in the central extended amygdala in mice. Neurosci Lett 457:58-60.

Seney ML, Cahill K, Enwright JF 3rd, Logan RW, Huo Z, Zong W, Tseng G, McClung CA (2019) Diurnal rhythms in gene expression in the prefrontal cortex in schizophrenia. Nat Commun 10:3355.

Shelton MA, Newman JT, Gu H, Sampson AR, Fish KN, MacDonald ML, Moyer CE, DiBitetto JV, Dorph-Petersen KA, Penzes P, Lewis DA, Sweet RA (2015) Loss of microtubule-associated protein 2 immunoreactivity linked to dendritic spine loss in schizophrenia. Biol Psychiatry 78:374-385.

Silva RH, Abílio VC, Takatsu AL, Kameda SR, Grassl C, Chehin AB, Medrano WA, Calzavara MB, Registro S, Andersen ML, Machado RB, Carvalho RC, Ribeiro Rde A, Tufik S, Frussa-Filho R (2004) Role of hippocampal oxidative stress in memory deficits induced by sleep deprivation in mice. Neuropharmacology 46:895-903.

Sims KS, Williams RS (1990) The human amygdaloid complex: a cytologic and histochemical atlas using Nissl, myelin, acetylcholinesterase and nicotinamide adenine dinucleotide phosphate diaphorase staining. Neuroscience 36:449-472.

Slaker M, Churchill L, Todd RP, Blacktop JM, Zuloaga DG, Raber J, Darling RA, Brown TE, Sorg BA (2015) Removal of perineuronal nets in the medial prefrontal cortex impairs the acquisition and reconsolidation of a cocaine-induced conditioned place preference memory. J Neurosci 35:4190-4202.

Slaker ML, Jorgensen ET, Hegarty DM, Liu X, Kong Y, Zhang F, Linhardt RJ, Brown TE, Aicher SA, Sorg BA (2018) Cocaine exposure modulates perineuronal nets and synaptic excitability of fastspiking interneurons in the medial prefrontal cortex. eNeuro 5: ENEURO.0221-18.2018.

Sorvari H, Soininen H, Paljärvi L, Karkola K, Pitkänen A (1995) Distribution of parvalbumin-immunoreactive cells and fibers in the human amygdaloid complex. J Comp Neurol 360:185-212.

Spano GM, Banningh SW, Marshall W, de Vivo L, Bellesi M, Loschky SS, Tononi G, Cirelli C (2019) Sleep deprivation by exposure to novel objects increases synapse density and axon-spine interface in the hippocampal CA1 region of adolescent mice. J Neurosci 39:6613-6625.

Steullet P, Cabungcal JH, Bukhari SA, Ardelt MI, Pantazopoulos H, Hamati F, Salt TE, Cuenod M, Do KQ, Berretta S (2018) The thalamic reticular nucleus in schizophrenia and bipolar disorder: role of parvalbumin-expressing neuron networks and oxidative stress. Mol Psychiatry 23:2057-2065.

Stevens B, Schafer DP (2018) Roles of microglia in nervous system development, plasticity, and disease. Dev Neurobiol 78:559-560.

Szklarczyk A, Lapinska J, Rylski M, McKay RD, Kaczmarek L (2002) Matrix metalloproteinase- 9 undergoes expression and activation during dendritic remodeling in adult hippocampus. J Neurosci 22:920-930.

Takayama F, Zhang X, Hayashi Y, Wu Z, Nakanishi H (2017) Dysfunction in diurnal synaptic responses and social behavior abnormalities in cathepsin S-deficient mice. Biochem Biophys Res Commun 490:447-452.

Tononi G, Cirelli C (2006) Sleep function and synaptic homeostasis. Sleep Med Rev 10:49-62.

Tononi G, Cirelli C (2014) Sleep and the price of plasticity: from synaptic and cellular homeostasis to memory consolidation and integration. Neuron 81:12-34.

Vyas A, Mitra R, Shankaranarayana Rao BS, Chattarji S (2002) Chronic stress induces contrasting patterns of dendritic remodeling in hippocampal and amygdaloid neurons. J Neurosci 22:68106818.

Wake H, Moorhouse AJ, Miyamoto A, Nabekura J (2013) Microglia: actively surveying and shaping neuronal circuit structure and function. Trends Neurosci 36:209-217.

Wang JL, Lim AS, Chiang WY, Hsieh WH, Lo MT, Schneider JA, Buchman AS, Bennett DA, Hu K, Saper CB (2015) Suprachiasmatic neuron numbers and rest-activity circadian rhythms in older humans. Ann Neurol 78:317-322.

Wells AM, Ridener E, Bourbonais CA, Kim W, Pantazopoulos H, Carroll FI, Kim KS, Cohen BM, Carlezon WA (2017) Effects of chronic social defeat stress on sleep and circadian rhythms are mitigated by kappa-opioid receptor antagonism. J Neurosci 37:7656-7668.

Wen TH, Afroz S, Reinhard SM, Palacios AR, Tapia K, Binder DK, Razak KA, Ethell IM (2018) Genetic reduction of matrix metalloproteinase- 9 promotes formation of perineuronal nets around parvalbumin-expressing interneurons and normalizes auditory cortex responses in developing Fmr1 knock-out mice. Cereb Cortex 28:3951-3964.

Xue YX, Xue LF, Liu JF, He J, Deng JH, Sun SC, Han HB, Luo YX, Xu LZ, Wu P, Lu L (2014) Depletion of perineuronal nets in the amygdala to enhance the erasure of drug memories. J Neurosci 34:6647-6658.

Yang S, Hilton S, Alves JN, Saksida LM, Bussey T, Matthews RT, Kitagawa H, Spillantini MG, Kwok JCF, Fawcett JW (2017) Antibody recognizing 4-sulfated chondroitin sulfate proteoglycans restores memory in tauopathy-induced neurodegeneration. Neurobiol Aging 59:197-209.

Zhou JN, Riemersma RF, Unmehopa UA, Hoogendijk WJ, van Heerikhuize JJ, Hofman MA, Swaab DF (2001) Alterations in arginine vasopressin neurons in the suprachiasmatic nucleus in depression. Arch Gen Psychiatry 58:655-662. 